Pre-feasibility evaluation for the generation of electrical energy through the synergy between wind energy and water pumping storage in San Cristóbal Island - Galápagos

\title{
Evaluación de la pre factibilidad para la generación de energía eléctrica mediante la sinergia entre las energías eólica y almacenamiento por bombeo de agua en la isla San Cristóbal - Galápagos
}

\author{
$\begin{array}{ll}\text { V. E. Burbano } & \\ & \text { R. A. Narváez } \\ & 2,3,1\end{array}$ \\ ${ }^{1}$ Universidad Internacional SEK, Quito, Ecuador \\ E-mail: vladimir.burbanov@gmail.com \\ ${ }^{2}$ Instituto de Investigación Geológico y Energético, Quito, Ecuador \\ E-mail: ricardo.narvaez@geoenergia.gob.ec \\ ${ }^{3}$ Universidad Central del Ecuador, UCE-GIIP, Quito, Ecuador \\ E-mail: ranarvaez@uce.edu.ec
}

Abstract

This document presents an investigation that aims to contribute to the mitigation of climate change, in addition to seeking energy autonomy based on Renewable Energy. For that reason, this research evaluates the prefeasibility of a renewable energy storage system based on water pumping on San Cristóbal - Galápagos Island, where there is a potential energy source located in El Junco lagoon. For this, the calculations were made based on the island's demand curve during 2018, taking into account that it is desired to cover the peak of such energy demand -at night that is between 16:00h and 22:00h-. Storage tanks are calculated according to the required volume, in addition to the characteristics that the generators may have to cover that peak demand. For the return of the water used in the generation, a pumping system is calculated based on the energy obtained through the wind resource of San Cristóbal, which corresponds to 3 wind turbines -of which its power curve is analyzed together with the average speed of the wind per hour during 2018-. The energy delivered by the wind turbines to carry out the required pumping is obtained, in addition to the required characteristics of the pumps. The results demonstrate that the proposal is viable given that the demand for the night peak is covered, considering that the energy used is purely renewable.
Resumen

Este documento expone una investigación que tiene como objetivo aportar a la mitigación del cambio climático, además de buscar autonomía energética basándose en Energías Renovables. Por tal motivo esta investigación evalúa la prefactibilidad de un sistema de almacenamiento de energía renovable basado en bombeo de agua en la isla San Cristóbal Galápagos, donde se tiene una fuente de energía almacenada debido a la diferencia de nivel disponible en la laguna El Junco. Se realizan los cálculos en base a la curva de demanda de la isla durante el año 2018, contando con que se desea cubrir el pico de dicha demanda de energía de la noche que se encuentra entre las $16 \mathrm{~h} 00$ y las $22 \mathrm{~h} 00$. Se calculan los tanques de almacenamiento de acuerdo al volumen requerido además de las características que deben tener los generadores de energía. Para el retorno del agua usada en la generación se calcula un sistema de bombeo en base a la energía obtenida por medio del recurso eólico de San Cristóbal, mismo que corresponde a 3 aerogeneradores de los cuales se analiza su curva de potencia, y junto con la velocidad promedio del viento por hora durante el año 2018, se obtiene la energía entregada por los aerogeneradores para realizar el bombeo requerido, además de las características de las bombas. Los resultados muestran que la propuesta es viable en vista de que se cubre la demanda del pico de la noche, considerando que la energía usada es netamente renovable.
Index terms- Hybrid system, wind, hydraulic, storage, electricity generation, pumped.
Palabras clave-- Sistema hibrido, eólica, hidráulica, almacenamiento, generación eléctrica, bombeo. 


\section{INTRODUCCIÓN}

Para mitigar los efectos provocados por las emisiones de gases de efecto invernadero se han buscado nuevas formas de obtención de energía donde no se requiera el uso de combustibles fósiles [1]. Para esto, se propone el aprovechamiento de las energías renovables o también llamadas energías limpias [2].

Más de 1500 millones de personas a nivel mundial aún carecen de acceso a energía eléctrica [3], la mayoría de estas personas viven en pequeños pueblos o islas remotas, los mismos que están aislados de los sistemas robustos de energía, Para estas zonas remotas los recursos son esenciales para lograr un resultado relevante en cuestión de energía, donde temas como la contaminación, el riesgo asociado al combustible, el transporte y almacenamiento, costos operativos excesivos, etc. [4], son importantes para el análisis de la oferta energética, por ejemplo, para islas pequeñas, los costos de los combustibles fósiles adquieren un valor bastante importante, alcanzando más del $15 \%$ de todas sus importaciones [5].

Además del alto costo del combustible y en general de las fuentes de energía fósiles [6], hay que tener en cuenta los efectos económicos que trae consigo el aumento de sus costos [5]. Adicional, este tipo de generación es un serio contaminante ambiental debido a la emisión de los gases de efecto invernadero que esta produce, además de que los recursos energéticos comunes, como el carbón, el petróleo y el gas natural, se están agotando muy rápidamente [2].

La Administración de Información de Energía de EE. UU. (EIA) realizó un estudio sobre el costo y el rendimiento de las nuevas tecnologías de generación de energía eléctrica a escala de servicios públicos [7]. Se hace una comparación entre los costos totales de Motores de Combustión Interna (MCI) y Aerogeneradores teniendo en cuenta la capacidad de la que se dispone de cada uno en San Cristóbal, en base a casos similares de la EIA. Los dos casos representan la instalación de capacidad pico o suplementaria para un municipio o pequeña empresa de servicios públicos [7].

El caso de MCI de $20 \mathrm{MW}$ de potencia, el costo base estimado para este caso de tecnología totaliza $\$ 1810 / \mathrm{kW}$ [7]. Asimismo, se detalla el caso de un proyecto eólico onshore con una capacidad total de $50 \mathrm{MW}$, considerada una planta pequeña, el costo base estimado para este caso de tecnología totaliza $\$ 1677 / \mathrm{kW}$ [7].

La comparación indica que para el mercado referencial que es el de Estados Unidos el costo de implementar MCI es mayor que el costo de implementar un parque eólico onshore de pequeña capacidad, además se debe considerar que el combustible para los MCI es gas natural, el mismo que es más económico que el diésel que actualmente se usa para alimentar los MCI de San Cristóbal.

\subsection{Oferta Energética en Zonas Remotas}

La prioridad absoluta de los sistemas como los que se encuentran en las regiones o zonas insulares, aislados de la zona continental, es la de satisfacer la demanda energética total [8], puesto que por lo general no existen sistemas de generación eléctrica robustos en dichas zonas. La generación por medio de motores de combustión interna es la principal fuente de energía eléctrica que abastece a una gran mayoría de áreas remotas en el mundo [4], además de ser una fuente confiable en relación a las energías renovables por la estabilidad que ofrece en la producción de energía eléctrica y que si se lo requiere puede generarse de manera constante mientras se disponga de combustible [6].

Desafortunadamente el uso de los motores diésel plantea enormes desafíos técnicos, financieros y sobre todo ambientales [4], esto debido a los fuertes incrementos en los costos de operación, mantenimiento y combustible de dichos equipos que a su vez son grandes fuentes de emisión de dióxido de carbono $\mathrm{CO} 2$, perjudiciales para el ambiente del planeta [9], por lo que en la actualidad, la sustitución de motores diésel por energías renovables se ha convertido en una prioridad [6].

\subsection{Naturaleza de las Energías Renovables}

El principal problema en el aprovechamiento de las energías renovables es su intermitencia en la generación de energía [10], debido a que dependen mucho de fenómenos naturales como por ejemplo el viento, el sol o las corrientes marinas, se sabe que dichos elementos no se encuentran presentes todo el tiempo en toda la superficie del planeta por lo que la intermitencia es una característica natural de las energías renovables [3]. La experiencia de Dinamarca demostró que los problemas de estabilidad ocurren cuando las tasas de penetración son superiores al $20 \%$ o $30 \%$ [11], esto es debido a que cuando más grande es la penetración del viento, mayor es la necesidad de regulación.

\subsection{Sistemas de Energía Híbridos}

Para lo antes mencionado, se busca el implemento de sistemas que mitiguen la contaminación ambiental [9], en la actualidad se están desarrollando sistemas que integran fuentes de energía renovable para que en un futuro no muy lejano se elimine o al menos se reduzca la dependencia que casi todos los países del mundo tienen respecto a los combustibles fósiles [5].

Los Sistemas de Energía Híbridos son la combinación de generación de energía con fuentes renovables y/o convencionales [2]. El uso de estos sistemas se da sobre todo en áreas rurales donde la demanda de energía no es muy alta [3], como por ejemplo en islas donde sus características geográficas dificultan y vuelven muy costosa la interconexión con otros sistemas de 
abastecimiento de energía robustos por lo tanto la implementación de Sistemas Híbridos de generación es una gran opción [13]. Con los sistemas de energía híbridos, la capacidad de las energías renovables puede aumentar significativamente en redes autónomas saturadas y puede también proporcionar confiabilidad a los sistemas sustituyendo los altos costos al momento de cubrir la demanda de energía en horas pico [14].

Los Sistemas híbridos de energías renovables son ideales para otorgar autonomía energética a sitios remotos [3], como por ejemplo a islas que se encuentran alejadas y son habitadas por personas. Esto, debido a que al encontrarse en locaciones remotas, no es fácil su conexión con sistemas más robustos de energía eléctrica ya que la interconexión resultaría altamente costosa [3], considerando que dichas redes se ubican por lo general en la superficie continental.

En la Tabla 1, se presenta un cuadro comparativo acerca de los sistemas híbridos investigados:

Tabla 1. Comparación de los sistemas híbridos investigados

\begin{tabular}{|c|c|c|c|}
\hline Ubicación & $\begin{array}{c}\text { Sistema } \\
\text { Híbrido }\end{array}$ & $\begin{array}{c}\text { Almacenamiento } \\
\text { por bombeo }\end{array}$ & Simulación \\
\hline $\begin{array}{c}\text { Tuktoyaktuk } \\
\text { - Northern } \\
\text { Canadá }\end{array}$ & $\begin{array}{c}\text { Diésel, eólica y } \\
\text { aire } \\
\text { comprimido }\end{array}$ & No & $\begin{array}{c}\text { MATLAB / } \\
\text { Simulink }\end{array}$ \\
\hline Creta - Grecia & $\begin{array}{c}\text { Diesel, eólica y } \\
\text { fotovoltáica }\end{array}$ & $\begin{array}{c}\text { Sí } \\
\text { (Proyeción) }\end{array}$ & Power World \\
\hline Hong Kong & $\begin{array}{c}\text { Eólica, } \\
\text { hidráulica }\end{array}$ & $\begin{array}{c}\text { Sí } \\
\text { (Proyeción) }\end{array}$ & HOMER \\
\hline $\begin{array}{c}\text { Isla Brava - } \\
\text { Cabo Verde }\end{array}$ & $\begin{array}{c}\text { Eólica, } \\
\text { hidráulica }\end{array}$ & Sí & $\begin{array}{c}\text { Simulación } \\
\text { computarizada }\end{array}$ \\
\hline $\begin{array}{c}\text { Ikaria - } \\
\text { Grecia }\end{array}$ & $\begin{array}{c}\text { Eólica, } \\
\text { hidráulica }\end{array}$ & $\begin{array}{c}\text { Algoritmo de } \\
\text { simulación por } \\
\text { (diagrama flujo) }\end{array}$ \\
\hline
\end{tabular}

La base de este estudio es el de analizar la factibilidad de que pueda haber para una posible implementación de este tipo de sistemas en lugares que por su historia, ubicación geográfica e importancia para el planeta son ideales, como por ejemplo la islas Galápagos [15], mismas que se diferencian de muchas islas a nivel mundial debido a que fueron declaradas en 1978 por la Organización de las Naciones Unidas para la Educación, la Ciencia y la Cultura (UNESCO) como Patrimonio Natural de la Humanidad [16].

\subsection{Sistemas de Almacenamiento de Energía}

El almacenamiento de energía hidroeléctrica bombeada es una tecnología bastante conocida y comercialmente aceptable [8], la misma que puede ser posteriormente transformada a electricidad.

Existen de dos tipos de almacenamiento hidráulico por bombeo, el primero hace referencia al almacenamiento de agua de mar y el segundo hace referencia al almacenamiento de agua dulce, la principal diferencia entre estos consiste en el reservorio inferior, que en el primer caso es el mar, el cual presenta un volumen ilimitado de agua [17], mientras que en el segundo caso se tiene un volumen bastante limitado que va de acuerdo a la capacidad de embalses o tanques. El bombeo y la eficiencia de generación es casi del $90 \%$, esto difiere de la tasa de eficiencia de otros sistemas hidráulicos debido a las pérdidas en las compuertas [11]. Los sistemas híbridos que incluyen el almacenamiento hidráulico por bombeo sirven para reemplazar al almacenamiento por baterías, que podrían contener plomo y ácido sulfúrico y ser perjudiciales para el ambiente [3].

\subsection{Laguna El Junco}

En la isla se encuentran un sitio de visita que es un icono de San Cristóbal llamado "El Junco" caracterizado por ser de uso público [18]. Esta laguna tiene un gran potencial de almacenamiento de energía ya que se ubica a una altura de $660 \mathrm{~m}$ sobre el nivel del mar y tiene una capacidad volumétrica de $360000 \mathrm{~m}^{3}$, esto debido a que su nivel se mantiene solamente por las aguas de lluvia y por la intensa neblina que casi constantemente la cubre, lo cual reduce la evaporación [19].

\subsection{Parque Eólico San Cristóbal}

En octubre de 2007 se inauguró el parque eólico de las Galápagos, en la isla San Cristóbal, con una potencia eólica instalada de 2,4 MW y una producción anual estimada de 3,2 GWh [20]. El Parque Eólico se encuentra ubicado en el Cerro El Tropezón, constituido por tres aerogeneradores de $800 \mathrm{~kW}$ [21].

El proyecto eólico fue financiado en un $80 \%$ por organismos internacionales, el G8 y el programa de las Naciones Unidas para el Desarrollo (UNDP), y en un $20 \%$ por Elecgalápagos y el municipio de San Cristóbal [20].

\section{METODOLOGÍA}

El procedimiento para la aplicación del diseño de experimentos requiere considerar las siguientes etapas:

1. Definir el objeto del análisis e identificar las variables que se requiere tratar.

2. Identificar los factores que potencialmente podrían influir en la función objetivo, y los valores que éstos pueden tomar. Entre estos valores se buscará la información necesaria.

3. Establecer una estrategia de análisis para cálculos.

4. Efectuar los cálculos con los valores de los factores decididos en el punto 2 para obtener los valores de las respuestas estudiadas.

5. Comprobar lo acertado de los cálculos realizados, caso contrario volver al paso 3 .

Para la evaluación de esta propuesta, se realiza el dimensionamiento de un sistema hidráulico para el transporte de agua, dicho sistema será utilizado para generación de energía eléctrica mediante turbogeneradores y para devolver el agua al reservorio por medio de bombeo. 
Al tener como tema la evaluación de prefactibilidad de un sistema, se han tomado en cuenta los cálculos con el criterio de efecto que tiene una o varias variables sobre uno o más factores que influyen directamente en dicha evaluación, dichos factores se encuentran en los puntos que se indican a continuación:

1. Análisis de la capacidad energética de San Cristóbal.

2. Dimensionamiento de Tanques para almacenamiento de agua.

3. Cálculo de los parámetros para generación.

4. Cálculo de los parámetros para bombeo.

5. Selección de la(s) bomba(s) para retorno de agua desde tanques hasta reservorio.

\subsection{Análisis de la Capacidad Energética de San Cristóbal}

En este ítem se analiza la información base que se emplea para el análisis del tema propuesto en este documento, misma que se detalla a continuación:

\subsubsection{Curva de potencia de los aerogeneradores}

En la Tabla 2 se muestran los valores de velocidad del viento y potencia. Dichos valores corresponden a la Curva de Potencia de los aerogeneradores ubicados en el parque eólico de San Cristóbal. Se emplea datos de 2018, considerando la disponibilidad basada en los registros históricos presentes al momento de la investigación.

Tabla 2: Datos para curva de Potencia Aerogeneradores

\begin{tabular}{|c|c|c|c|}
\hline $\begin{array}{c}\text { Velocidad } \\
\text { Viento }[\mathbf{m} / \mathbf{s}]\end{array}$ & $\begin{array}{c}\text { Potencia } \\
{[\mathbf{k W}]}\end{array}$ & $\begin{array}{c}\text { Velocidad } \\
\text { Viento }[\mathbf{m} / \mathbf{s}]\end{array}$ & $\begin{array}{c}\text { Potencia } \\
{[\mathbf{k W}]}\end{array}$ \\
\hline 4 & 25 & 15 & 800 \\
\hline 5 & 73 & 16 & 800 \\
\hline 6 & 144 & 17 & 800 \\
\hline 7 & 239 & 18 & 800 \\
\hline 8 & 359 & 19 & 800 \\
\hline 9 & 510 & 20 & 800 \\
\hline 10 & 649 & 21 & 800 \\
\hline 11 & 769 & 22 & 800 \\
\hline 12 & 800 & 23 & 800 \\
\hline 13 & 800 & 24 & 800 \\
\hline 14 & 800 & 25 & 800 \\
\hline
\end{tabular}

* Datos 2018

\subsubsection{Velocidad del viento en San Cristóbal}

La Tabla 3 muestra el promedio de la velocidad del viento en San Cristóbal por cada hora del día durante el año 2018.
Tabla 3: Velocidad promedio de viento en San Cristóbal

\begin{tabular}{|c|c|c|c|}
\hline $\begin{array}{c}\text { Tiempo } \\
\text { [horas] }\end{array}$ & $\begin{array}{c}\text { Velocidad } \\
\text { promedio } \\
\text { viento [m/s] }\end{array}$ & $\begin{array}{c}\text { Tiempo } \\
\text { [horas] }\end{array}$ & $\begin{array}{c}\text { Velocidad } \\
\text { promedio } \\
\text { viento [m/s] }\end{array}$ \\
\hline $1: 00$ & 6,3787 & $13: 00$ & 6,3786 \\
\hline $2: 00$ & 6,3788 & $14: 00$ & 6,3783 \\
\hline $3: 00$ & 6,3789 & $15: 00$ & 6,3781 \\
\hline $4: 00$ & 6,3790 & $16: 00$ & 6,3780 \\
\hline $5: 00$ & 6,3791 & $17: 00$ & 6,3779 \\
\hline $6: 00$ & 6,3793 & $18: 00$ & 6,3779 \\
\hline $7: 00$ & 6,3795 & $19: 00$ & 6,3777 \\
\hline $8: 00$ & 6,3796 & $20: 00$ & 6,3774 \\
\hline $9: 00$ & 6,3795 & $21: 00$ & 6,3769 \\
\hline $10: 00$ & 6,3794 & $22: 00$ & 6,3764 \\
\hline $11: 00$ & 6,3792 & $23: 00$ & 6,3759 \\
\hline $12: 00$ & 6,3789 & $0: 00$ & 6,3786 \\
\hline * Datos 2018 & & \multicolumn{3}{l}{}
\end{tabular}

\subsubsection{Generación del parque eólico en San Cristóbal}

La Tabla 4 muestra los promedios de la generación eólica de la isla San Cristóbal. La generación neta indica el promedio de producción diaria total durante 2018.

Tabla 4: Promedios de generación eólica de la isla San Cristóbal

\begin{tabular}{|c|c|c|c|}
\hline $\begin{array}{c}\text { Tiempo } \\
\text { [horas] }\end{array}$ & $\begin{array}{c}\text { Generación } \\
\text { neta }[\mathbf{k W}]\end{array}$ & $\begin{array}{c}\text { Tiempo } \\
{[\text { horas] }}\end{array}$ & $\begin{array}{c}\text { Generación } \\
\text { neta [kW] }\end{array}$ \\
\hline $1: 00$ & 288,48 & $13: 00$ & 305,86 \\
\hline $2: 00$ & 302,14 & $14: 00$ & 302,42 \\
\hline $3: 00$ & 291,95 & $15: 00$ & 297,78 \\
\hline $4: 00$ & 294,34 & $16: 00$ & 279,24 \\
\hline $5: 00$ & 283,90 & $17: 00$ & 283,27 \\
\hline $6: 00$ & 290,92 & $18: 00$ & 287,57 \\
\hline $7: 00$ & 275,26 & $19: 00$ & 288,72 \\
\hline $8: 00$ & 292,99 & $20: 00$ & 273,69 \\
\hline $9: 00$ & 287,81 & $21: 00$ & 285,66 \\
\hline $10: 00$ & 299,83 & $22: 00$ & 280,49 \\
\hline $11: 00$ & 304,94 & $23: 00$ & 279,77 \\
\hline $12: 00$ & 298,98 & $0: 00$ & 275,52 \\
\hline Datos 2018 & \multicolumn{3}{l}{} \\
\hline
\end{tabular}

\subsubsection{Generación neta total en San Cristóbal}

La Tabla 5 muestra el promedio de generación horaria neta total de la isla San Cristóbal durante 2018.

Tabla 5: Generación neta total promedio de la isla San Cristóbal

\begin{tabular}{|c|c|c|c|}
\hline $\begin{array}{c}\text { Tiempo } \\
\text { [horas] }\end{array}$ & $\begin{array}{c}\text { Generación neta } \\
\text { total [kW] }\end{array}$ & $\begin{array}{c}\text { Tiempo } \\
\text { [horas] }\end{array}$ & $\begin{array}{c}\text { Generación neta } \\
\text { total [kW] }\end{array}$ \\
\hline $1: 00$ & 1477,70 & $13: 00$ & 2025,62 \\
\hline $2: 00$ & 1402,97 & $14: 00$ & 2058,25 \\
\hline $3: 00$ & 1363,43 & $15: 00$ & 2048,59 \\
\hline $4: 00$ & 1341,41 & $16: 00$ & 2037,15 \\
\hline $5: 00$ & 1339,90 & $17: 00$ & 1939,17 \\
\hline $6: 00$ & 1370,68 & $18: 00$ & 1937,36 \\
\hline $7: 00$ & 1458,02 & $19: 00$ & 2265,81 \\
\hline $8: 00$ & 1666,35 & $20: 00$ & 2165,61 \\
\hline $9: 00$ & 1826,75 & $21: 00$ & 2067,75 \\
\hline $10: 00$ & 1912,14 & $22: 00$ & 1916,86 \\
\hline $11: 00$ & 1994,21 & $23: 00$ & 1752,24 \\
\hline $12: 00$ & 2013,95 & $0: 00$ & 1616,54 \\
\hline
\end{tabular}

\subsection{Dimensionamiento de Tanques para Almacenamiento de Agua}

Los cálculos para el dimensionamiento de los tanques se plantean en base a la energía requerida para cubrir la 
demanda del pico de la noche, y asumiendo una altura de $8 \mathrm{~m}$ para que no afecte de manera sustancial el paisaje de la zona, se obtiene el volumen que se requiere de agua para lograr generar la energía eléctrica requerida en horas pico de demanda para la población de la isla San Cristóbal-Galápagos.

Para el dimensionamiento de tanques se tiene como condiciones iniciales las siguientes:

- La energía requerida en las horas pico de demanda para la isla.

- Las dimensiones que puede tener un tanque sin afectar visualmente el entorno.

Esto para lograr tener el volumen necesario para suplir la demanda del horario pico en la isla.

Se realiza el cálculo con dos diferentes fórmulas, la primera se refiere al tanque con diámetro y altura iguales mediante la ecuación (1) mientras que la segunda refiere al cálculo del diámetro con una altura máxima propuesta mediante la ecuación (2).

$$
\begin{aligned}
& D 1_{\text {tanque }}=\sqrt[3]{\frac{4 * V}{\pi}} \\
& D 2_{\text {tanque }}=\sqrt{\frac{4 * V}{\pi * h}}
\end{aligned}
$$

\subsection{Cálculo de los Parámetros para Generación}

Para la generación se tienen como condiciones iniciales las siguientes:

- La energía requerida en las horas pico de demanda para la isla.

- Capacidad de generación del generador a seleccionar en base a la potencia requerida.

- Caudal requerido para alcanzar la capacidad de generación.

- Pérdidas del sistema de tuberías.

Para el análisis de la generación se hace uso de la ecuación de la energía (3), que contempla los siguientes términos como indican Mott y Robert L, en su libro Mecánica de Fluidos:

- $\mathrm{hA}=$ Energía que se agrega al fluido con un dispositivo mecánico, como una bomba; es frecuente que se le denomine carga total sobre la bomba [22].

- $\mathrm{hR}=$ Energía que se remueve del fluido por medio de un dispositivo mecánico, como un motor de fluido [22].

- $\mathrm{hL}=$ Pérdidas de energía del sistema por la fricción en las tuberías, o pérdidas menores por válvulas y otros accesorios [22].

$$
\frac{P_{1}}{\mathrm{Y}}+h_{1}+\frac{V_{1}{ }^{2}}{2 g}-h_{R}-h_{L}+h_{A}=\frac{P_{2}}{Y}+h_{2}+\frac{V_{2}{ }^{2}}{2 g}
$$

Donde se tienen las siguientes consideraciones:
- Superficie del depósito expuesta a la atmósfera entonces $\mathrm{P}_{1}=0$.

- Corriente libre de fluido expuesta a la atmósfera entonces $\mathrm{P}_{2}=0$.

- Aproximadamente el área superficial del depósito es grande entonces $\mathrm{V}_{1}=0$.

- Sin elementos que agreguen energía al fluido entonces $\mathrm{hA}=0$.

Por lo que al despejar hR se tiene (4), además se obtiene hL mediante la ecuación de Darcy (5) para pérdidas de energía por fricción en tuberías. El factor de fricción se obtiene por medio de (6), la potencia que es capaz de entregar el fluido a las turbinas se obtiene con (7) y las pérdidas menores se obtienen con (8).

$$
\begin{gathered}
h_{R}=\left(h_{1}-h_{2}\right)-\frac{V_{2}{ }^{2}}{2 g}-h_{L} \\
h_{L}=f * \frac{L_{T}}{D} * \frac{V_{2}{ }^{2}}{2 g} \\
f=\frac{0,25}{\left[\log \left(\frac{1}{3,7 *\left(\frac{D}{\varepsilon}\right)}+\frac{5,74}{R e^{0,9}}\right)\right]^{2}} \\
P_{R}=h_{R} * Y * Q \\
h_{\text {Lmenores }}=K * \frac{V_{2}^{2}}{2 g}
\end{gathered}
$$

\subsection{Cálculo de los Parámetros para Bombeo}

Para el bombeo se tienen como condiciones iniciales las siguientes:

- Diámetro de la tubería.

- Pérdidas del sistema de tuberías.

Para el análisis respecto al bombeo se hace uso de la ecuación de la energía (3), donde se tienen las siguientes consideraciones:

- Superficie del depósito expuesta a la atmósfera entonces $\mathrm{P}_{1}=0$.

- Corriente libre de fluido expuesta a la atmósfera entonces $\mathrm{P}_{2}=0$.

- Aproximadamente el área superficial del depósito es grande entonces $\mathrm{V}_{1}=0$.

- Sin elementos que reciban energía del fluido entonces $\mathrm{hR}=0$.

Por lo que al despejar hA se tiene (9), además se obtiene hL mediante la ecuación de Darcy (5) para pérdidas de energía por fricción en tuberías. El factor de fricción se obtiene por medio de (6), la potencia que requiere la bomba se obtiene con (10) y las pérdidas menores se obtienen con (8).

$$
\begin{gathered}
\boldsymbol{h}_{\boldsymbol{A}}=\left(\boldsymbol{h}_{\mathbf{2}}-\boldsymbol{h}_{1}\right)+\frac{{\boldsymbol{V}_{2}}^{2}}{2 g}+\boldsymbol{h}_{L} \\
\boldsymbol{P}_{\boldsymbol{A}}=\boldsymbol{h}_{\boldsymbol{A}} * Y * \boldsymbol{Q}
\end{gathered}
$$




\subsection{Selección de la(s) Bomba(s) para Retorno de Agua desde Tanques hasta Reservorio}

Para la selección de la(s) bomba(s) se tienen en cuenta los siguientes parámetros:

- Los cálculos obtenidos respecto a bombeo.

- Análisis de los parámetros propios de bombas.

Para la obtención de la curva resistente del sistema se parte de (11) que es la ecuación de Bernoulli, entre los puntos A y C, ubicados en la superficie del líquido en los depósitos de aspiración y de descarga, respectivamente [23], donde A corresponde a los tanques de almacenamiento y $\mathrm{C}$ a la Laguna El Junco.

Los términos $\mathrm{H}_{\mathrm{B} 1}, \mathrm{H}_{\mathrm{B} 2}$, etc., se incluyen en la ecuación dependiendo del número de bombas que se coloquen en serie.

$$
H_{A}-\Delta H_{A-C}+\left(H_{B 1}+H_{B 2}+\cdots+H_{B n}\right)=H_{C}
$$

Donde $s=$ succión e i=impulsión.

$$
\begin{gathered}
\boldsymbol{Z}_{A}+\frac{\boldsymbol{P}_{A}}{\mathrm{Y}}+\frac{\boldsymbol{\alpha} * V_{A}{ }^{2}}{2 g}-\left(\boldsymbol{h}_{f s}-\sum \boldsymbol{h}_{L s}\right)-\left(\boldsymbol{h}_{f i}-\sum \boldsymbol{h}_{L i}\right)+ \\
\left(\boldsymbol{H}_{B 1}+\boldsymbol{H}_{B 2}+\cdots+\boldsymbol{H}_{B n}\right)=\boldsymbol{Z}_{C}+\frac{\boldsymbol{P}_{C}}{\mathrm{Y}}+\frac{\boldsymbol{\alpha} * V_{C}{ }^{2}}{2 \boldsymbol{g}}
\end{gathered}
$$

$P_{A}=0 \quad$ y $\quad P_{C}=0$ debido a que están a presión atmosférica.

$V_{A}=0$ y $V_{C}=0$ debido a que el área de superficie de los almacenamientos es grande.

$$
\begin{gathered}
\left(\boldsymbol{Z}_{C}-\boldsymbol{Z}_{\boldsymbol{A}}\right)+\left(\boldsymbol{h}_{f s}+\boldsymbol{h}_{f i}\right)+\left(\sum \boldsymbol{h}_{L s}+\sum \boldsymbol{h}_{L i}\right)=\boldsymbol{H}_{B 1}+\boldsymbol{H}_{B 2}+ \\
\cdots+\boldsymbol{H}_{B n}
\end{gathered}
$$

Las pérdidas de carga por rozamiento se calculan con la ecuación de Darcy \& Weisbach [23]:

$$
h_{f}=\frac{8 * f * L * Q_{T}^{2}}{\pi^{2} * g * D^{5}}
$$

Las pérdidas de carga localizadas, debidas a válvulas y accesorios, se calcularán con la ecuación de Borda \& Carnot [23]:

$$
\boldsymbol{h}_{L}=\boldsymbol{k}_{L} * \frac{V^{2}}{2 * g}=\frac{8 * \sum k_{L} * Q_{T}{ }^{2}}{\pi^{2} * g * D^{4}}
$$

Se reemplazan (14) y (15) en (16):

$$
\begin{gathered}
\left(Z_{C}-Z_{A}\right)+\left(\frac{8 * f_{s} * L_{s} * Q_{T}{ }^{2}}{\pi^{2} * g^{*} D_{s}{ }^{5}}+\frac{8 * f_{i} * L_{i} * Q_{T}{ }^{2}}{\pi^{2} * g * D_{i}{ }^{5}}\right)+\left(\frac{8 * \sum k_{L s} * Q_{T}{ }^{2}}{\pi^{2} * g * D_{s}{ }^{4}}+\right. \\
\left.\frac{8 * \sum k_{L i} * Q_{T}{ }^{2}}{\pi^{2} * g * D_{i}{ }^{4}}\right)=H_{B 1}+H_{B 2}+\cdots+H_{B n}
\end{gathered}
$$

Se tratan los términos para reducir (16):

$$
\begin{gathered}
\left(Z_{C}-Z_{A}\right)+\frac{8}{\pi^{2} * g} *\left[\frac{f *\left(L_{S}+L_{i}\right)}{D^{5}}+\frac{\sum k_{L s}+\sum k_{L i}}{D^{4}}\right] * Q_{T}{ }^{2}=H_{B 1}+ \\
H_{B 2}+\cdots+H_{B n}
\end{gathered}
$$

Se obtiene entonces (18) que es la curva resistente del sistema:

$$
\boldsymbol{H}_{\boldsymbol{r}}=\left(\boldsymbol{Z}_{\boldsymbol{C}}-\boldsymbol{Z}_{\boldsymbol{A}}\right)+\frac{\mathbf{8}}{\boldsymbol{\pi}^{2} \boldsymbol{g}^{*} \boldsymbol{D}_{\boldsymbol{s}}{ }^{5}} *\left(\boldsymbol{f} * \boldsymbol{L}_{\boldsymbol{T}}+\boldsymbol{D} * \sum \boldsymbol{k}_{L T}\right) * \boldsymbol{Q}_{\boldsymbol{T}}{ }^{2}
$$

Se obtiene también (19) que es la curva motriz correspondiente a la(s) bomba(s) para dicho sistema.

$$
H_{m}=H_{B 1}+H_{B 2}+\cdots+H_{B n}
$$

Para esta investigación se considera el uso de una bomba o, de dos o más bombas colocadas en serie debido a que se requiere ganar altura.

En serie, la tubería de impulsión de una bomba se constituye en la tubería de aspiración de la siguiente unidad, por lo que el caudal bombeado, $\mathrm{Q}_{\mathrm{T}}$, es el mismo para todas ellas, y la altura del conjunto es la suma de las alturas desarrolladas por las bombas individualmente [23].

Por otra parte, la(s) bomba(s) contemplada(s) tiene(n) su correspondiente ecuación característica como lo indica (20), donde $\mathrm{N}$ es el término que representa al número de bombas en serie para el sistema, sin embargo (20) es válida únicamente cuando las bombas en serie tienen las mismas características técnicas.

$$
\boldsymbol{H}_{\boldsymbol{m}}=\boldsymbol{N} *\left(\boldsymbol{A}+\boldsymbol{B} * \boldsymbol{Q}_{T}+\boldsymbol{C} *{\boldsymbol{Q}_{T}}^{2}\right)
$$

\section{RESULTADOS Y DISCUSIÓN}

La Fig. 1 muestra el trayecto de la tubería que va desde la laguna El Junco hacia el poblado de San Cristóbal, dicha tubería cubre una distancia de $9600 \mathrm{~m}$ y bordea la carretera que va desde el poblado de San Cristóbal y pasa por las faldas del cerro donde se encuentra la laguna. Además, se indica también la elevación de la superficie terrestre que va desde los 320 $\mathrm{m}$ hasta los $660 \mathrm{~m}$.

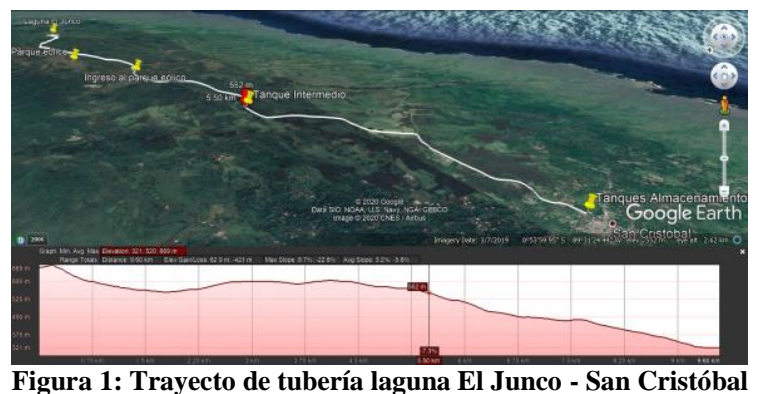

En la Fig. 2 se muestra la curva de potencia de los aerogeneradores en color naranja y en color gris la curva del polinomio de quinto orden, dicha curva es la que más se acerca a la curva de potencia de los aerogeneradores y de la misma se obtiene la ecuación (21).

$$
\begin{gathered}
P=-0.0062 * V^{5}+0.3853 * V^{4}-8.4529 * V^{3}+ \\
73.926 * V^{2}-146.72 * V+112.14
\end{gathered}
$$

La ecuación (21) permite obtener la generación eólica esperada que se observa en la Tabla 1, reemplazando los valores de velocidad de viento por cada hora durante el año 2018 . 


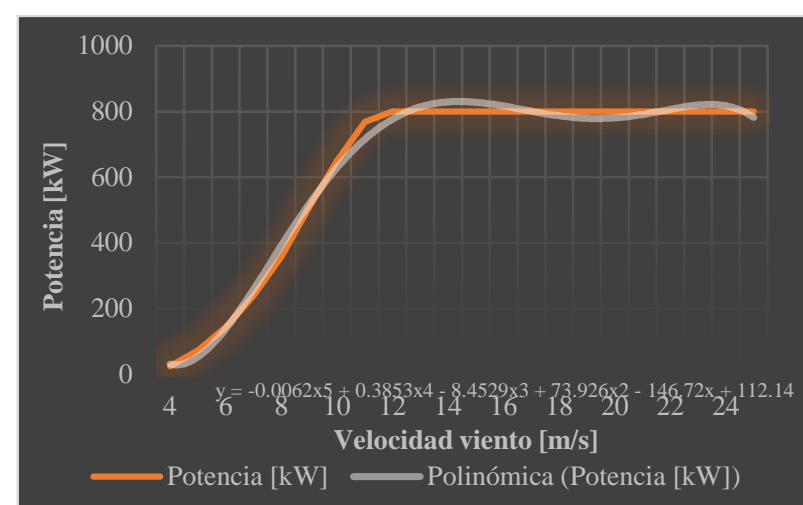

Figura 2: Curva de Potencia Aerogeneradores

La Tabla 6 muestra los promedios respecto a la generación eólica de la isla San Cristóbal, la generación neta indica el promedio de producción total de energía de un día por hora durante el año 2018 de los aerogeneradores, la Generación esperada es el promedio de la producción posible en base a la curva de potencia de los aerogeneradores y a la velocidad de viento en las zona del parque eólico, mientras que la Generación perdida es la diferencia de las dos anteriores y que representa un promedio de energía que se está desaprovechando en cuanto a generación eólica que corresponde a $30843,67 \mathrm{~kW}$.

Tabla 6: Promedios de generación eólica de la isla San Cristóbal

\begin{tabular}{|c|c|c|c|}
\hline $\begin{array}{c}\text { Tiempo } \\
\text { [horas] }\end{array}$ & $\begin{array}{c}\text { Generación } \\
\text { neta [kW] }\end{array}$ & $\begin{array}{c}\text { Generación } \\
\text { esperada [kW] }\end{array}$ & $\begin{array}{c}\text { Generación } \\
\text { perdida [kW] }\end{array}$ \\
\hline $1: 00$ & 288,48 & 1574,94 & 1286,46 \\
\hline $2: 00$ & 302,14 & 1574,98 & 1272,84 \\
\hline $3: 00$ & 291,95 & 1575,01 & 1283,06 \\
\hline $4: 00$ & 294,34 & 1575,05 & 1280,71 \\
\hline $5: 00$ & 283,90 & 1575,08 & 1291,18 \\
\hline $6: 00$ & 290,92 & 1575,14 & 1284,22 \\
\hline $7: 00$ & 275,26 & 1575,18 & 1299,93 \\
\hline $8: 00$ & 292,99 & 1575,20 & 1282,21 \\
\hline $9: 00$ & 287,81 & 1575,18 & 1287,38 \\
\hline $10: 00$ & 299,83 & 1575,14 & 1275,31 \\
\hline $11: 00$ & 304,94 & 1575,07 & 1270,13 \\
\hline $12: 00$ & 298,98 & 1574,98 & 1276,00 \\
\hline $13: 00$ & 305,86 & 1574,85 & 1268,99 \\
\hline $14: 00$ & 302,42 & 1574,76 & 1272,34 \\
\hline $15: 00$ & 297,78 & 1574,69 & 1276,90 \\
\hline $16: 00$ & 279,24 & 1574,63 & 1295,39 \\
\hline $17: 00$ & 283,27 & 1574,62 & 1291,35 \\
\hline $18: 00$ & 287,57 & 1574,59 & 1287,03 \\
\hline $19: 00$ & 288,72 & 1574,54 & 1285,82 \\
\hline $20: 00$ & 273,69 & 1574,41 & 1300,72 \\
\hline $21: 00$ & 285,66 & 1574,24 & 1288,58 \\
\hline $22: 00$ & 280,49 & 1574,08 & 1293,59 \\
\hline $23: 00$ & 279,77 & 1573,90 & 1294,13 \\
\hline $0: 00$ & 275,52 & 1574,91 & 1299,39 \\
\hline
\end{tabular}

En la Fig. 3 se ven representados los datos de la Tabla 5 y la Tabla 6.

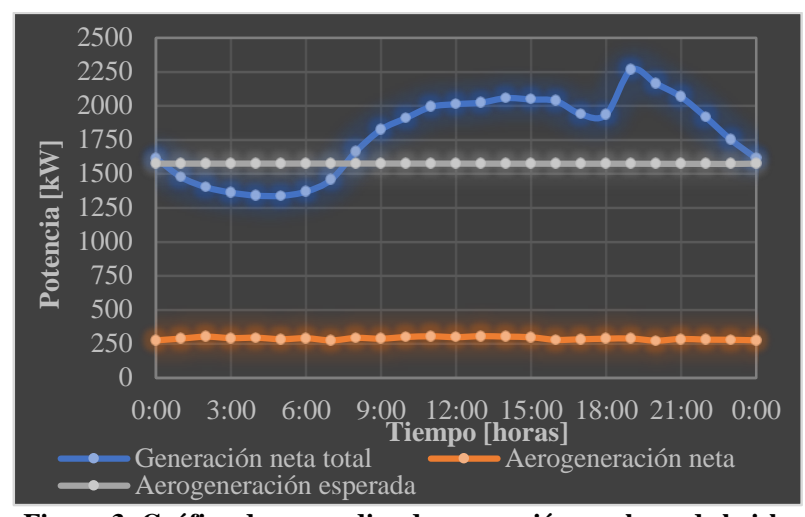

Figura 3: Gráfica de promedios de generación por hora de la isla San Cristóbal

\subsection{Dimensionamiento de Tanques para Almacenamiento de Agua}

En la Fig. 4 mediante el uso de software CAD se obtiene el área bajo la curva que corresponde a la energía promedio pico de la isla San Cristóbal en horas de la noche entre las 18:00h y 22:00h, y el área es de 7,1545 $\mathrm{cm}^{2}$ que corresponde a 715,45 kWh de energía, además esta área se obtiene de la curva que hace referencia a la generación neta total de la Fig. 3.

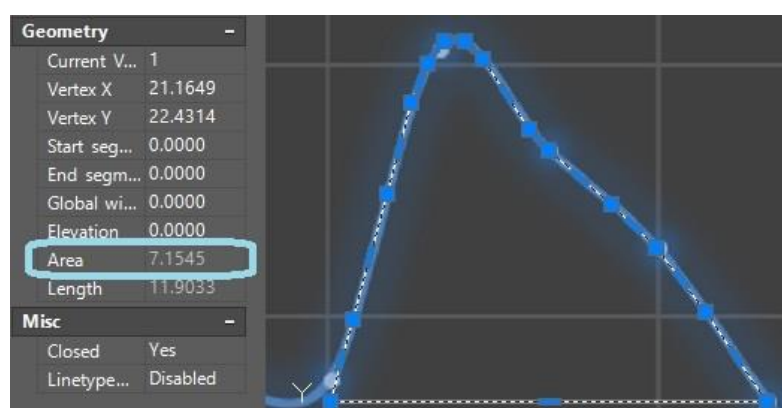

Figura 4: Energía promedio pico de la noche entre las 18:00h y 22:00h

El resultado de energía necesaria para generación durante el horario de 18:00h a 22:00h que indica la Tabla 7 es de 715,45 kWh.

Tabla 7: Energía necesaria para generación

\begin{tabular}{|c|c|c|}
\hline $\begin{array}{c}\text { Area obtenida } \\
{\left[\mathbf{c m}^{2}\right]}\end{array}$ & $\begin{array}{c}\text { Factor de } \\
\text { conversión }\end{array}$ & $\begin{array}{c}\text { Energía para generar } \\
\text { por 4 horas [kWh] }\end{array}$ \\
\hline 7,15 & 100 & 715,45 \\
\hline
\end{tabular}

En la Tabla 8 se muestra la potencia promedio mínima por hora que se requiere para generar la suficiente energía para suplir el pico de la noche y que corresponde a 0,179 MW.

Tabla 8: Potencia promedio mínima por hora para generadores

\begin{tabular}{|c|c|c|}
\hline Descripción & Cantidad & Unidad \\
\hline Energía & 715,450 & $\mathrm{kWh}$ \\
\hline $\begin{array}{c}\text { Energía pico noche 18:00 a 22:00 } \\
\text { (4 horas) }\end{array}$ & 0,715 & $\mathrm{MWh}$ \\
\cline { 2 - 3 } & 25419,393 & $\mathrm{~atm}^{*} \mathrm{~m}^{3}$ \\
\hline Potencia promedio hora pico noche & 0,179 & $\mathrm{MW}$ \\
\hline
\end{tabular}

En la Tabla 9 se muestran los resultados referentes al cálculo de los tanques de almacenamiento que se necesitan para la generación de la energía requerida para 
cubrir el pico de demanda de la noche que muestra la Fig. 4, para este cálculo se consideran tanques cilíndricos para almacenamiento de agua.

Tabla 9: Cálculo de tanques de almacenamiento

\begin{tabular}{|c|c|c|}
\hline Descripción & Cantidad & Unidad \\
\hline Distancia & 9600 & $\mathrm{~m}$ \\
\hline Diferencia de altura & 340 & $\mathrm{~m}$ \\
\hline Presión hidrostática disponible & 32,914 & $\mathrm{~atm}$ \\
\hline Volumen total & 772,301 & $\mathrm{~m}^{3}$ \\
\hline Diámetro tanque & 15,679 & $\mathrm{~m}$ \\
\hline \# de tanques & 2 & $\mathrm{u}$ \\
\hline Volumen por tanque & 386,150 & $\mathrm{~m}^{3}$ \\
\hline Cálculo donde altura tanque $=$ diámetro tanque \\
\hline Diámetro 1 tanque unidad & 7,893 & $\mathrm{~m}$ \\
\hline Cálculo con altura tanque & 8 & $\mathrm{~m}$ \\
\hline Diámetro 2 tanque unidad & 7,839 & $\mathrm{~m}$ \\
\hline
\end{tabular}

Con el volumen requerido para generación, se realiza el cálculo de la cantidad de tanques para almacenamiento del agua, que en este caso es de 2 unidades de almacenamiento que pueden ser de 7,89 $\mathrm{m}$ tanto de altura como de diámetro o de $8 \mathrm{~m}$ de altura y 7,84 $\mathrm{m}$ de diámetro.

\subsection{Generación}

Para el análisis de generación se hace uso del método indicado en el punto 2.3. Cálculo de los Parámetros para Generación.

La Tabla 10 indica la potencia que será transmitida a las turbinas hidráulicas sin contar con las pérdidas menores, con un caudal mínimo de $0,085 \mathrm{~m}^{3} / \mathrm{s}$, dicho caudal se propone para lograr obtener todos los parámetros de dicha tabla, como se observa el número de Reynolds corresponde a un régimen turbulento, el factor de fricción obtenido por el diagrama de Moody [22], en el caso de la generación, es igual al obtenido con (5) y corresponde a 0,018 con lo cual se tiene las pérdidas de energía más representativas que son hL igual a 39,318 m y hR igual a $300,613 \mathrm{~m}$ para de esta forma obtener mediante (6) una potencia transmitida por el agua de $0,250 \mathrm{MW}$.

Tabla 10: Potencia disponible para las turbinas hidráulicas sin

pérdidas menores
\begin{tabular}{|c|c|c|}
\hline Descripción & Cantidad & Unidad \\
\hline Caudal mínimo Darcy & 0,085 & $\mathrm{~m}^{3} / \mathrm{s}$ \\
\hline Velocidad & 1,165 & $\mathrm{~m} / \mathrm{s}$ \\
\hline Reynolds (Régimen turbulento) & 376048,277 & - \\
\hline Factor fricción (f) diagrama Moody & 0,0180 & - \\
\hline Factor fricción (f) fórmula & 0,0180 & - \\
\hline Pérdidas debido a la fricción (hL) & 39,318 & $\mathrm{~m}$ \\
\hline Energía entregada a la turbina (hR) & 300,613 & $\mathrm{~m}$ \\
\hline Potencia transmitida por el agua & 0,250 & $\mathrm{MW}$ \\
\hline
\end{tabular}

En la Tabla 11 Se muestran las pérdidas menores por accesorios en el sistema de tuberías, se han considerado el mayor número posible de codos tanto de $45^{\circ}$ como de $90^{\circ}$ y el máximo número de válvulas de compuerta que son las que más altas pérdidas producen en un sistema en relación a otros tipos de válvulas, se calcula con la ecuación (7) donde $\mathrm{K}$ tiene un valor distinto por cada accesorio, el total de pérdidas menores de energía es de 0,010 MWh.

Tabla 11: Pérdidas menores de energía

\begin{tabular}{|c|c|c|}
\hline Descripción & Cantidad & Unidad \\
\hline V2/2g=K entrada & 0,069 & $\mathrm{~m}$ \\
\hline Promedio máximo \# codos $45^{\circ}$ & 200 & $\mathrm{u}$ \\
\hline Promedio máximo \# codos $90^{\circ}$ & 50 & $\mathrm{u}$ \\
\hline $\begin{array}{c}\text { Promedio máximo \# válvulas de } \\
\text { compuerta }\end{array}$ & 50 & $\mathrm{u}$ \\
\hline Pérdidas en codos $45^{\circ}$ & 2,883 & $\mathrm{~m}$ \\
\hline Pérdidas en codos $90^{\circ}$ & 1,351 & $\mathrm{~m}$ \\
\hline Pérdidas válvulas de compuerta & 0,360 & $\mathrm{~m}$ \\
\hline & 4,663 & $\mathbf{m}$ \\
\cline { 2 - 3 } & 0,451 & $\mathrm{~atm}$ \\
\cline { 2 - 3 } & 348,647 & $\mathrm{~atm} \mathrm{~m}^{3}$ \\
\cline { 2 - 3 } Total pérdidas menores & 35326621,114 & $\mathrm{Joules}^{\circ}$ \\
\cline { 2 - 3 } & 0,010 & $\mathrm{MWh}$ \\
\hline
\end{tabular}

En la Tabla 12 se muestran los datos de la potencia propuesta de 0,250 MW para cada turbina considerando un $90 \%$ de eficiencia que tienen las turbomáquinas modernas y un porcentaje de reserva para generación correspondiente a $20,5 \%$.

Tabla 12: Potencia para turbinas hidráulicas

\begin{tabular}{|c|c|c|}
\hline Descripción & Cantidad & Unidad \\
\hline Potencia promedio hora para pico noche & 0,179 & $\mathrm{MW}$ \\
\hline Turbinas de agua & 4 & $\mathrm{u}$ \\
\hline Potencia que requeriría cada turbina & 0,045 & $\mathrm{MW}$ \\
\hline $\begin{array}{c}\text { Potencia transmitida por el agua menos } \\
\text { pérdidas menores }\end{array}$ & 0,240 & $\mathrm{MW}$ \\
\hline \% mínimo a reducir & 25,53 & $\%$ \\
\hline Potencia propuesta para cada turbina & 0,250 & $\mathrm{MW}$ \\
\hline $\begin{array}{c}\text { Potencia con eficiencia de 90\% para } \\
\text { turbomáquinas modernas }\end{array}$ & 0,225 & $\mathrm{MW}$ \\
\hline Porcentaje de reserva para generación & 20,5 & $\%$ \\
\hline
\end{tabular}

\subsection{Bombeo}

Para el bombeo se consideran 3 etapas, una inicial, una intermedia y otra final. La primera etapa analizada es la intermedia debido a que es la principal y donde más se aprovecha la energía disponible para bombear, misma que es obtenida de los aerogeneradores. Las otras dos etapas se proponen para bombear el faltante de volumen que se indica en la Tabla 16.

\subsubsection{Bombeo intermedio}

En la Fig. 5 se observa una parte de la Fig. 3 que corresponde a la energía disponible para bombeo en el horario de $02: 10 \mathrm{~h}$ a $06: 20 \mathrm{~h}$, mediante el uso de software CAD se obtiene el área del rectángulo señalado que representa a la energía que podría ser aprovechada para realizar el bombeo desde los tanques de almacenamiento hacia el reservorio natural en la laguna el Junco, su valor es de $8,291 \mathrm{~cm}^{2}$, en el eje Y se representa a la energía y cada $1 \mathrm{~cm}$ corresponde a $100 \mathrm{~kW}$, mientras que en el eje $\mathrm{X}$ se representa al tiempo y cada $1 \mathrm{~cm}$ corresponde a 1 hora, como se muestra en la Tabla 13. 


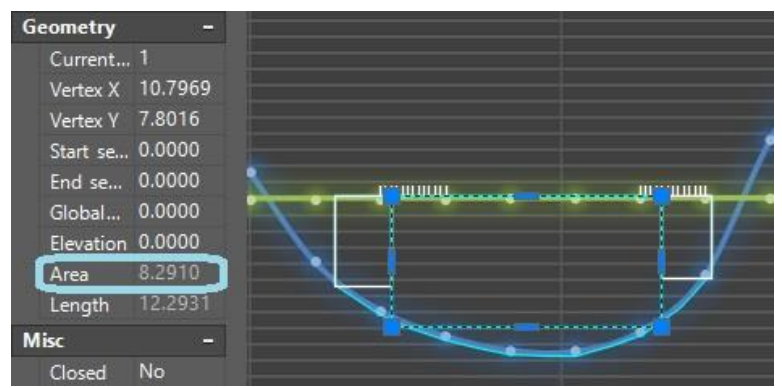

Figura 5: Energía para bombeo intermedio

El resultado de energía disponible para bombeo durante el horario de 02:10h a 06:20h que indica la Tabla 13 es de 829,10 kWh.

Tabla 13: Energía disponible para bombeo intermedio

\begin{tabular}{|c|c|c|}
\hline $\begin{array}{c}\text { Area obtenida } \\
{\left[\mathbf{c m}^{\mathbf{2}}\right]}\end{array}$ & $\begin{array}{c}\text { Factor de } \\
\text { conversión }\end{array}$ & $\begin{array}{c}\text { Energía para bombeo } \\
\text { durante 4:10 horas [kWh] }\end{array}$ \\
\hline 8,291 & 100 & 829,100 \\
\hline
\end{tabular}

Para el análisis de bombeo se hace uso del método indicado en el punto 2.4. Cálculo de los Parámetros para Bombeo.

En la Tabla 14 se muestran las pérdidas menores por accesorios en el sistema de tuberías, se han considerado el mayor número posible de codos tanto de $45^{\circ}$ como de $90^{\circ}$ y el máximo número de válvulas de compuerta que son las que más altas pérdidas producen en un sistema en relación a otros tipos de válvulas, se calcula con la ecuación (8) donde $\mathrm{K}$ tiene un valor distinto por cada accesorio, el total de pérdidas menores es de 1,466 m.

Tabla 14: Pérdidas menores de energía en bombeo intermedio

\begin{tabular}{|c|c|c|}
\hline Descripción & Cantidad & Unidad \\
\hline V2/2g=K entrada & 0,022 & $\mathrm{~m}$ \\
\hline Promedio máximo \# codos $45^{\circ}$ & 200 & $\mathrm{u}$ \\
\hline Promedio máximo \# codos $90^{\circ}$ & 50 & $\mathrm{u}$ \\
\hline $\begin{array}{c}\text { Promedio máximo \# válvulas de } \\
\text { compuerta }\end{array}$ & 50 & $\mathrm{u}$ \\
\hline Pérdida en codos $45^{\circ}$ & 0,906 & $\mathrm{~m}$ \\
\hline Pérdida en codos $90^{\circ}$ & 0,425 & $\mathrm{~m}$ \\
\hline Pérdida válvulas de compuerta & 0,113 & $\mathrm{~m}$ \\
\hline Total pérdidas menores & 1,466 & $\mathrm{~m}$ \\
\hline
\end{tabular}

La Tabla 15 indica las pérdidas totales de energía en bombeo intermedio, con un caudal calculado de 0,048 $\mathrm{m}^{3} / \mathrm{s}$, como se observa el número de Reynolds corresponde a un régimen turbulento, el factor de fricción obtenido por el diagrama de Moody [22], en este caso para bombeo, es igual al obtenido con (5) y corresponde a 0,0188 con lo cual se tiene las pérdidas de energía más representativas que son por fricción en la tubería $(\mathrm{hL})$ igual a $12,857 \mathrm{~m}$ y hA igual a $352,879 \mathrm{~m}$ para de esta forma obtener mediante (10) una potencia requerida total para bombear de $164300,687 \mathrm{~W}$. Mientras que las pérdidas totales para el bombeo intermedio corresponden a $30,128 \mathrm{kWh}$.
Tabla 15: Pérdidas totales de energía en bombeo intermedio

\begin{tabular}{|c|c|c|}
\hline Descripción & Cantidad & Unidad \\
\hline Caudal & 0,048 & $\mathrm{~m}^{3} / \mathrm{s}$ \\
\hline Velocidad & 0,652 & $\mathrm{~m} / \mathrm{s}$ \\
\hline $\begin{array}{l}\text { Reynolds (Régimen } \\
\text { turbulento) }\end{array}$ & 210843,273 & - \\
\hline Factor de fricción (f) Moody & 0,0188 & - \\
\hline Factor de fricción (f) fórmula & 0,0188 & - \\
\hline Logitud de tuberia (LT) & 9600 & $\mathrm{~m}$ \\
\hline Altura 2 (h2) & 660 & $\mathrm{~m}$ \\
\hline Altura 1 (h1) & 320 & $\mathrm{~m}$ \\
\hline Pérdida en tubería (hL) & 12,903 & $\mathrm{~m}$ \\
\hline $\begin{array}{c}\text { Energía transferida al agua } \\
(\mathrm{hA})\end{array}$ & 352,925 & $\mathrm{~m}$ \\
\hline $\begin{array}{l}\text { Energía transferida al agua } \\
(\mathrm{hA})+\text { pérdidas menores }\end{array}$ & 353,067 & $\mathrm{~m}$ \\
\hline $\begin{array}{c}\text { Potencia requerida de la } \\
\text { bomba }\end{array}$ & 164629,790 & $\mathrm{~W}$ \\
\hline \multirow{6}{*}{ Total de Pérdidas } & 14,369 & $\mathrm{~m}$ \\
\hline & 1,391 & atm \\
\hline & 1074,277 & atm $* m^{3}$ \\
\hline & 108851106,944 & Joules \\
\hline & 0,030 & MWh \\
\hline & 30,236 & $\mathrm{kWh}$ \\
\hline
\end{tabular}

La Tabla 16 los parámetros de la bomba y del bombeo intermedio, donde se observa que la energía total disponible para el bombeo intermedio es de 2502040 736,958 Joules, mismo que otorga una capacidad para bombear $714,869 \mathrm{~m}^{3}$, al calcular la diferencia entre el volumen total de los tanques de almacenamiento y la capacidad de volumen bombeado, indicados en la Tabla 9 , se tiene que hay un volumen faltante por bombear de $57,432 \mathrm{~m}^{3}$, por lo que se agregan otras dos etapas de bombeo, una inicial que aprovecha la energía disponible antes del bombeo intermedio y otra final que aprovecha la energía disponible después del bombeo intermedio.

Se observa también en la Tabla 16 que la bomba cumple los parámetros necesarios para bombeo, con una eficiencia de $87 \%$, se requieren 11 bombas colocadas en serie y se observa que la altura proporcionada por las bombas supera a la altura que se requiere vencer, además cumple con que la potencia real de la bomba es superior al cálculo de la potencia requerida de la bomba.

Tabla 16: Parámetros de bomba y bombeo intermedio

\begin{tabular}{|c|c|c|}
\hline Descripción & Cantidad & Unidad \\
\hline $\begin{array}{c}\text { Energía disponible para } \\
\text { bombeo }\end{array}$ & 829,100 & $\mathrm{kWh}$ \\
\hline $\begin{array}{c}\text { Energía disponible para } \\
\text { bombeo - pérdidas totales }\end{array}$ & 798,864 & $\mathrm{kWh}$ \\
\hline Eficiencia de la bomba & $87 \%$ & - \\
\hline $\begin{array}{l}\text { Energía total disponible para } \\
\text { bombeo }\end{array}$ & 2502040736,958 & Joules \\
\hline Volumen máximo a bombear & 714,869 & $\mathrm{~m}^{3}$ \\
\hline Volumen faltante por bombear & 57,432 & $\mathrm{~m}^{3}$ \\
\hline Tiempo de bombeo & 4,167 & horas \\
\hline Caudal necesario para bombeo & 0,048 & $\mathrm{~m}^{3} / \mathrm{s}$ \\
\hline Altura a vencer & 340 & $\mathrm{~m}$ \\
\hline $\begin{array}{l}\text { Potencia necesaria para } \\
\text { bombeo }\end{array}$ & 223,686 & HP \\
\hline $\begin{array}{l}\text { altura proporcionada por la } \\
\text { bomba }\end{array}$ & 32 & $\mathrm{~m}$ \\
\hline \# bombas & 11 & $\mathrm{u}$ \\
\hline $\begin{array}{c}\text { Altura proporcionada por las } \\
\text { bombas }\end{array}$ & 352 & $\mathrm{~m}$ \\
\hline Potencia requerida de la bomba & 20,335 & $\mathrm{HP}$ \\
\hline Potencia real de la bomba & 25 & $\mathrm{HP}$ \\
\hline
\end{tabular}


La Fig. 6 muestra la curva característica de la bomba, así como la ecuación cuadrática (22), misma que es del tipo de la ecuación (20) con un $\mathrm{N}=1$.

$$
H_{m}=34,419+0.0433 * Q_{T}+3 E-04 * Q_{T}{ }^{2}
$$

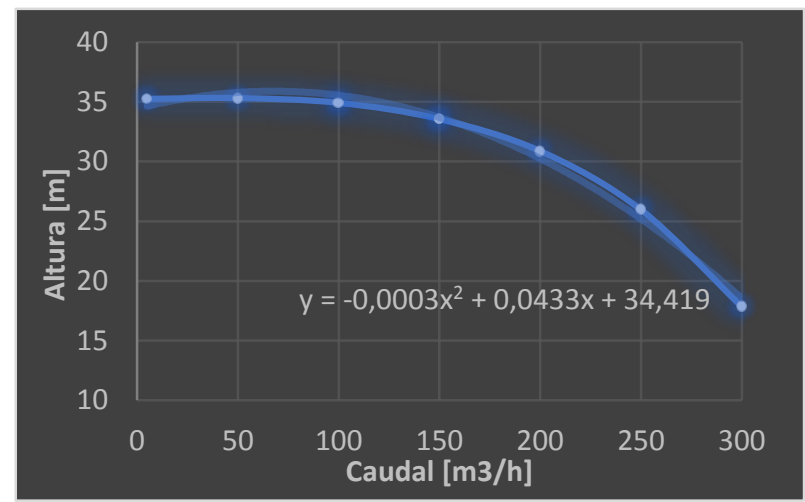

Figura 6: Curva característica de la bomba

Para la obtención de la ecuación de la curva resistente del sistema se utiliza el método mostrado en el punto 2.5. Selección de la(s) Bomba(s) para Retorno de Agua desde Tanques hasta Reservorio, de donde se reemplazan los datos obtenidos dejando (18) en función de $\mathrm{Q}_{\mathrm{T}}$, como se indica en (23).

$$
H_{r}=4,56 E-4 * Q_{T}^{2}+34
$$

Para la obtención de la ecuación de la curva motriz de la bomba se considera el caudal calculado en la Tabla 16, se realiza la búsqueda de la bomba que cumpla con dicho parámetro y que a su vez tenga una eficiencia capaz de cumplir con el bombeo en el rango de tiempo propuesto.

En la Metodología, en el punto 2.5. Selección de la(s) Bomba(s) para Retorno de Agua desde Tanques hasta Reservorio, se tiene el procedimiento para obtener la ecuación de la(s) bomba(s), de donde se obtienen los datos que se indican en la Tabla 17 , correspondientes al bombeo intermedio.

Tabla 17: Datos ecuaciones del sistema de bombeo intermedio

\begin{tabular}{|c|c|c|c|c|c|}
\hline $\begin{array}{c}\mathbf{Q} \\
{\left[\mathbf{m}^{\mathbf{3}} / \mathbf{h}\right]}\end{array}$ & $\mathbf{H r}[\mathbf{m}]$ & $\mathbf{H m}[\mathbf{m}]$ & $\begin{array}{c}\mathbf{Q} \\
{\left[\mathbf{m}^{\mathbf{3}} / \mathbf{h}\right]}\end{array}$ & $\mathbf{H r}[\mathbf{m}]$ & $\mathbf{H m}[\mathbf{m}]$ \\
\hline 0 & 340,000 & 378,609 & 125 & 347,008 & 386,584 \\
\hline 25 & 340,280 & 388,454 & 150 & 350,092 & 375,804 \\
\hline 50 & 341,121 & 394,174 & 175 & 353,736 & 360,899 \\
\hline 75 & 342,523 & 395,769 & 200 & 357,941 & 341,869 \\
\hline 100 & 344,485 & 393,239 & & & \\
\hline
\end{tabular}

La ecuación (24) se obtiene de multiplicar el número de bombas que corresponde a 11, tal como indica la Tabla 16 , por la ecuación (22).

$$
H_{m}=-3,3 E-3 * Q_{T}{ }^{2}+0,4763 * Q_{T}+378.61
$$

En la Tabla 18 se indican los datos del punto de funcionamiento $(\mathrm{PF})$, mismo que corresponde a $\left(\mathrm{Q}_{\mathrm{T}} ; \mathrm{H}_{\mathrm{m}}\right)$, por lo tanto, el PF es $(181,365 ; 356,447)$.
Tabla 18: Punto de funcionamiento del bombeo intermedio

\begin{tabular}{|c|c|c|}
\hline Descripción & Cantidad & Unidad \\
\hline $\mathrm{Q}_{\mathrm{T}}$ & 181,365 & $\mathrm{~m}^{3} / \mathrm{h}$ \\
\hline $\mathrm{Hr}$ & 356,447 & $\mathrm{~m}$ \\
\hline $\mathrm{Hm}$ & 356,447 & $\mathrm{~m}$ \\
\hline $\mathrm{Hr}-\mathrm{Hm}$ & 0,000 & $\mathrm{~m}$ \\
\hline
\end{tabular}

Como se indica en la Tabla 16 existe un volumen faltante por bombear que corresponde a $57,432 \mathrm{~m}^{3}$, por lo que se contempla un tanque intermedio. La Tabla 19 muestra la ubicación del tanque a lo largo del trayecto de tubería, con una capacidad de $58 \mathrm{~m}^{3}$ y los respectivos diámetros calculados, mismos que redondeando sus valores hacia arriba se obtiene un tanque cilíndrico de 5 $\mathrm{m}$ de diámetro y $5 \mathrm{~m}$ de altura.

\begin{tabular}{|} 
Tabla 19: Parámetros tanque intermedio \\
\begin{tabular}{|c|c|c|}
\hline Descripción & Cantidad & Unidad \\
\hline $\begin{array}{c}\text { Distancia entre tanques principales y } \\
\text { tanque intermedio }\end{array}$ & 4100 & $\mathrm{~m}$ \\
\hline $\begin{array}{c}\text { Distancia entre tanque intermedio y } \\
\text { laguna El Junco }\end{array}$ & 5500 & $\mathrm{~m}$ \\
\hline Diferencia de altura & 230 & $\mathrm{~m}$ \\
\hline Volumen total & 58 & $\mathrm{~m}^{3}$ \\
\hline Diámetro tanque & 4,297 & $\mathrm{~m}$ \\
\hline \# de tanques & 1 & $\mathrm{u}$ \\
\hline Volumen por tanque & 58 & $\mathrm{~m}^{3}$ \\
\hline Cálculo donde altura tanque $=$ diámetro tanque \\
\hline Diámetro tanque unidad & 4,195 & $\mathrm{~m}$ \\
\hline Cálculo con altura tanque & 4 & $\mathrm{~m}$ \\
\hline Diámetro tanque unidad & 4,297 & $\mathrm{~m}$ \\
\hline
\end{tabular}
\end{tabular}

\subsubsection{Bombeo inicial}

En la Fig. 7 se observa la energía disponible para bombeo en el horario de 01:20h a 02:10h, mediante el uso de software $\mathrm{CAD}$ se obtiene el área de el rectángulo señalado que representa a la energía que podría ser aprovechada para realizar el bombeo desde los tanques de almacenamiento principales hacia un tanque de almacenamiento intermedio, dicha área es de $1,2043 \mathrm{~cm}^{2}$, en el eje $\mathrm{Y}$ se representa a la energía y cada $1 \mathrm{~cm}$ corresponde a $100 \mathrm{~kW}$, mientras que en el eje $\mathrm{X}$ se representa al tiempo y cada $1 \mathrm{~cm}$ corresponde a 1 hora, como se muestra en la Tabla 20.

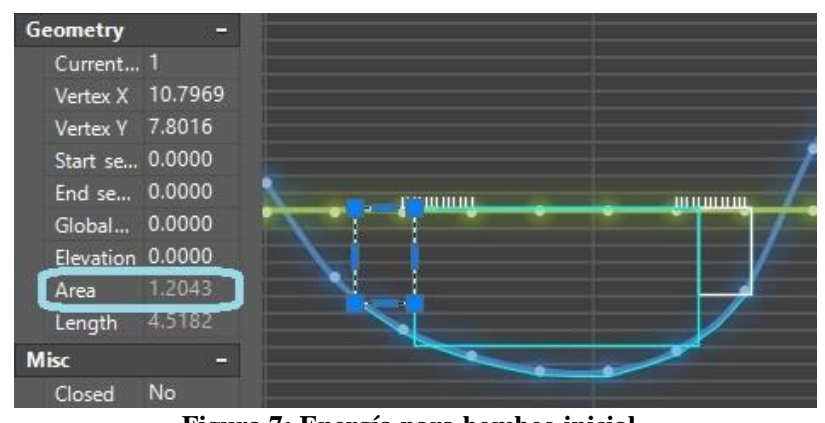

Figura 7: Energía para bombeo inicial

El resultado de energía disponible para bombeo durante el horario de 01:20h a 02:10h que indica la Tabla 20 es de 120,43 kWh. 
Tabla 20: Energía disponible para bombeo inicial

\begin{tabular}{|c|c|c|}
\hline $\begin{array}{c}\text { Area obtenida } \\
{\left[\mathbf{c m}^{2}\right]}\end{array}$ & $\begin{array}{c}\text { Factor de } \\
\text { conversión }\end{array}$ & $\begin{array}{c}\text { Energía para bombeo } \\
\text { durante 0:50 horas }[\mathbf{k W h}]\end{array}$ \\
\hline 1,204 & 100 & 120,43 \\
\hline
\end{tabular}

Para el análisis de bombeo se hace uso del método indicado en el punto 2.4. Cálculo de los Parámetros para Bombeo.

En la Tabla 21 se muestran las pérdidas menores por accesorios en el sistema de tuberías, donde $\mathrm{K}$ tiene un valor distinto por cada accesorio, el total de pérdidas menores es de $0,276 \mathrm{~m}$.

Tabla 21: Pérdidas menores de energía en bombeo inicial

\begin{tabular}{|c|c|c|}
\hline Descripción & Cantidad & Unidad \\
\hline V2/2g=K entrada & 0,006 & $\mathrm{~m}$ \\
\hline Promedio máximo \# codos $45^{\circ}$ & 150 & $\mathrm{u}$ \\
\hline Promedio máximo \# codos $90^{\circ}$ & 36 & $\mathrm{u}$ \\
\hline $\begin{array}{c}\text { Promedio máximo \# válvulas de } \\
\text { compuerta }\end{array}$ & 36 & $\mathrm{u}$ \\
\hline Pérdida en codos $45^{\circ}$ & 0,172 & $\mathrm{~m}$ \\
\hline Pérdida en codos $90^{\circ}$ & 0,078 & $\mathrm{~m}$ \\
\hline Pérdida válvulas de compuerta & 0,021 & $\mathrm{~m}$ \\
\hline Total pérdidas menores & 0,276 & $\mathrm{~m}$ \\
\hline
\end{tabular}

La Tabla 22 indica las pérdidas totales de energía en bombeo intermedio, con un caudal calculado de 0,024 $\mathrm{m}^{3} / \mathrm{s}$, como se observa el número de Reynolds corresponde a un régimen turbulento, el factor de fricción obtenido por el diagrama de Moody [22], en este caso para bombeo, es igual al obtenido con (5) y corresponde a 0,0202 con lo cual se tiene las pérdidas de energía más representativas que son $\mathrm{hL}$ igual a 1,504 $\mathrm{m}$ y hA igual a 231,509 m para de esta forma obtener mediante (10) una potencia requerida total para bombear de $54376,689 \mathrm{~W}$. Mientras que las pérdidas totales para el bombeo intermedio corresponden a $3,745 \mathrm{kWh}$.

Tabla 22: Pérdidas totales de energía en bombeo inicial

\begin{tabular}{|c|c|c|}
\hline Descripción & Cantidad & Unidad \\
\hline Caudal & 0,024 & $\mathrm{~m}^{3} / \mathrm{s}$ \\
\hline Velocidad & 0,329 & $\mathrm{~m} / \mathrm{s}$ \\
\hline Reynolds (Régimen turbulento) & 106194,611 & - \\
\hline Factor de fricción (f) diagrama \\
Moody & 0,0202 & - \\
\hline Factor de fricción (f) fórmula & 0,0202 & - \\
\hline Logitud de tuberia (LT) & 4100 & $\mathrm{~m}$ \\
\hline Altura 2 (h2) & 550 & $\mathrm{~m}$ \\
\hline Altura 1 (h1) & 320 & $\mathrm{~m}$ \\
\hline Pérdida en tubería (hL) & 1,504 & $\mathrm{~m}$ \\
\hline Energía transferida al agua (hA) & 231,509 & $\mathrm{~m}$ \\
\hline Energía transferida al agua (hA) + & 231,536 & $\mathrm{~m}$ \\
\hline pérdidas menores & 54376,689 & $\mathrm{~W}$ \\
\hline Potencia requerida de la bomba & 1,780 & $\mathrm{~m}$ \\
\hline & 0,172 & $\mathrm{~atm}$ \\
\cline { 2 - 3 } & 133,073 & $\mathrm{~atm}$ \\
\hline \multirow{2}{*}{ Total de Pérdidas } & \\
\cline { 2 - 3 } & 13483624,887 & $\mathrm{Joules}$ \\
\cline { 2 - 3 } & 0,004 & $\mathrm{MWh}$ \\
\cline { 2 - 3 } & 3,745 & $\mathrm{kWh}$ \\
\hline
\end{tabular}

La Tabla 23 muestra los parámetros de la bomba y del bombeo inicial, donde se observa que la energía total disponible para el bombeo inicial es de 252038 625,068 Joules, mismo que otorga una capacidad para bombear
$72,011 \mathrm{~m}^{3}$, al calcular la diferencia entre el volumen total de los tanques de almacenamiento que se observa en la Tabla 9 y la capacidad de volumen bombeado se tiene que existe un volumen sobrante de $14,579 \mathrm{~m}^{3}$, por lo que el bombeo inicial cubre la demanda de bombear el volumen faltante por bombear de la Tabla 16.

Se observa también en la Tabla 23 que la bomba cumple los parámetros necesarios para bombeo, con una eficiencia de $60 \%$, se requieren 7 bombas colocadas en serie y se observa que la altura proporcionada por las bombas supera a la altura que se requiere vencer, además cumple con que la potencia real de la bomba es superior al cálculo de la potencia requerida de la bomba.

Tabla 23: Parámetros de bomba y bombeo inicial

\begin{tabular}{|c|c|c|}
\hline Descripción & Cantidad & Unidad \\
\hline Energía disponible para bombeo & 120,430 & $\mathrm{kWh}$ \\
\hline $\begin{array}{c}\text { Energía disponible para bombeo } \\
\text { - pérdidas totales }\end{array}$ & 116,685 & $\mathrm{kWh}$ \\
\hline Eficiencia de la bomba & $60 \%$ & \\
\hline $\begin{array}{l}\text { Energía total disponible para } \\
\text { bombeo }\end{array}$ & 252038625,068 & Joules \\
\hline Volumen máximo a bombear & 72,011 & $\mathrm{~m}^{3}$ \\
\hline Volumen faltante por bombear & $-14,579$ & $\mathrm{~m}^{3}$ \\
\hline Tiempo de bombeo & 0,833 & horas \\
\hline Caudal necesario para bombeo & 0,024 & $\mathrm{~m}^{3} / \mathrm{s}$ \\
\hline Altura a vencer & 230 & $\mathrm{~m}$ \\
\hline Potencia necesaria para bombeo & 112,663 & $\mathrm{HP}$ \\
\hline $\begin{array}{c}\text { Altura proporcionada por la } \\
\text { bomba }\end{array}$ & 35 & $\mathrm{~m}$ \\
\hline \# bombas & 7 & $\mathrm{u}$ \\
\hline $\begin{array}{l}\text { Altura proporcionada por las } \\
\text { bombas }\end{array}$ & 245 & $\mathrm{~m}$ \\
\hline Potencia requerida de la bomba & 16,095 & HP \\
\hline Potencia real de la bomba & 25 & HP \\
\hline
\end{tabular}

Para la obtención de la ecuación de la curva resistente del sistema se utiliza el método mostrado en el punto 2.5. Selección de la(s) Bomba(s) para Retorno de Agua desde Tanques hasta Reservorio, de donde se reemplazan los datos obtenidos dejando (18) en función de $\mathrm{Q}_{\mathrm{T}}$, como se indica en (25).

$$
H_{r}=2,02 E-4 * Q_{T}{ }^{2}+230
$$

Para la obtención de la ecuación de la curva motriz de la bomba se considera el caudal calculado en la Tabla 22.

En la Metodología, en el punto 2.5. Selección de la(s) Bomba(s) para Retorno de Agua desde Tanques hasta Reservorio, se tiene el procedimiento para obtener la ecuación de la(s) bomba(s), de donde se obtienen los datos que se indican en la Tabla 24, correspondientes al bombeo inicial.

Tabla 24: Datos ecuaciones del sistema de bombeo inicial

\begin{tabular}{|c|c|c|c|c|c|}
\hline $\begin{array}{c}\mathbf{Q} \\
{\left[\mathbf{m}^{\mathbf{3}} / \mathbf{h}\right]}\end{array}$ & Hr $[\mathbf{m}]$ & $\mathbf{H m}[\mathbf{m}]$ & $\begin{array}{c}\mathbf{Q} \\
{\left[\mathbf{m}^{\mathbf{3}} / \mathbf{h}\right]}\end{array}$ & Hr $[\mathbf{m}]$ & Hm $[\mathbf{m}]$ \\
\hline 0 & 230,000 & 240,933 & 125 & 233,164 & 246,008 \\
\hline 25 & 230,127 & 247,198 & 150 & 234,556 & 239,148 \\
\hline 50 & 230,506 & 250,838 & 175 & 236,201 & 229,663 \\
\hline 75 & 231,139 & 251,853 & 200 & 238,099 & 217,553 \\
\hline 100 & 232,025 & 250,243 & & & \\
\hline
\end{tabular}


La ecuación (26) se obtiene de multiplicar el número de bombas que corresponde a 7 , tal como indica la Tabla 23, por la ecuación (22).

$H_{m}=-2,1 E-3 * Q_{T}^{2}-0,3031 * Q_{T}+240,93$

En la Tabla 25 se indican los datos del punto de funcionamiento, mismo que corresponde a $\left(\mathrm{Q}_{\mathrm{T}} ; \mathrm{H}_{\mathrm{m}}\right)$, por lo tanto, el PF es $(161,253 ; 235,201)$.

Tabla 25: Punto de funcionamiento del bombeo inicial

\begin{tabular}{|c|c|c|}
\hline Descripción & Cantidad & Unidad \\
\hline $\mathrm{QT}$ & 161,253 & $\mathrm{~m}^{3} / \mathrm{h}$ \\
\hline $\mathrm{Hr}$ & 235,201 & $\mathrm{~m}$ \\
\hline $\mathrm{Hm}$ & 235,201 & $\mathrm{~m}$ \\
\hline $\mathrm{Hr}-\mathrm{Hm}$ & 0,000 & $\mathrm{~m}$ \\
\hline
\end{tabular}

\subsubsection{Bombeo final}

En la Fig. 8 se observa la energía disponible para bombeo en el horario de 06:20h a 07:05h, mediante el uso de software CAD se obtiene el área de el rectángulo señalado que representa a la energía que podría ser aprovechada para realizar el bombeo desde el tanque de almacenamiento intermedio hasta la laguna El Junco, dicha área es de $0,967 \mathrm{~cm}^{2}$, en el eje Y se representa a la energía y cada $1 \mathrm{~cm}$ corresponde a $100 \mathrm{~kW}$, mientras que en el eje $X$ se representa al tiempo y cada $1 \mathrm{~cm}$ corresponde a 1 hora, como se muestra en la Tabla 26.

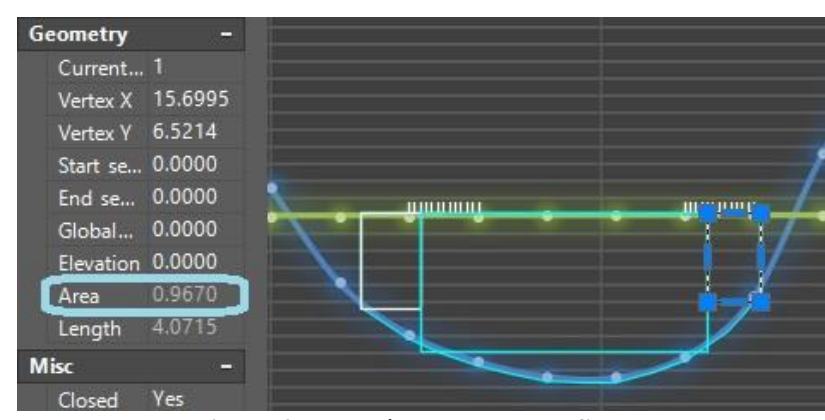

Figura 8: Energía para bombeo final

El resultado de energía disponible para bombeo durante el horario de 06:20h a 07:05 h que indica la Tabla 26 es de 96,7 kWh.

Tabla 26: Energía disponible para bombeo final

\begin{tabular}{|c|c|c|}
\hline $\begin{array}{c}\text { Area obtenida } \\
{\left[\mathbf{c m}^{2}\right]}\end{array}$ & $\begin{array}{c}\text { Factor de } \\
\text { conversión }\end{array}$ & $\begin{array}{c}\text { Energía para bombeo } \\
\text { durante 0:45 horas [kWh] }\end{array}$ \\
\hline 0,967 & 100 & 96,7 \\
\hline
\end{tabular}

Para el análisis de bombeo se hace uso del método indicado en el punto 2.4. Cálculo de los Parámetros para Bombeo.

En la Tabla 27 se muestran las pérdidas menores por accesorios en el sistema de tuberías, donde $\mathrm{K}$ tiene un valor distinto por cada accesorio, el total de pérdidas menores es de $0,084 \mathrm{~m}$.
Tabla 27: Pérdidas menores de energía en bombeo final

\begin{tabular}{|c|c|c|}
\hline Descripción & Cantidad & Unidad \\
\hline V2/2g=K entrada & 0,004 & $\mathrm{~m}$ \\
\hline Promedio máximo \# codos $45^{\circ}$ & 50 & $\mathrm{u}$ \\
\hline Promedio máximo \# codos $90^{\circ}$ & 16 & $\mathrm{u}$ \\
\hline $\begin{array}{c}\text { Promedio máximo \# válvulas de } \\
\text { compuerta }\end{array}$ & 16 & $\mathrm{u}$ \\
\hline Pérdida en codos $45^{\circ}$ & 0,045 & $\mathrm{~m}$ \\
\hline Pérdida en codos $90^{\circ}$ & 0,027 & $\mathrm{~m}$ \\
\hline Pérdida válvulas de compuerta & 0,007 & $\mathrm{~m}$ \\
\hline Total pérdidas menores & 0,084 & $\mathrm{~m}$ \\
\hline
\end{tabular}

La Tabla 28 indica las pérdidas totales de energía en bombeo final, con un caudal calculado de $0,021 \mathrm{~m}^{3} / \mathrm{s}$, como se observa el número de Reynolds corresponde a un régimen turbulento, el factor de fricción obtenido por el diagrama de Moody [22], en este caso para bombeo, es igual al obtenido con (5) y corresponde a 0,0205 con lo cual se tiene las pérdidas de energía más representativas que son hL igual a 1,611 $\mathrm{m}$ y hA igual a $111,616 \mathrm{~m}$ para de esta forma obtener mediante (10) una potencia requerida total para bombear de 23 248,682 W. Mientras que las pérdidas totales para el bombeo intermedio corresponden a $3,567 \mathrm{kWh}$.

Tabla 28: Pérdidas totales de energía en bombeo inicial

\begin{tabular}{|c|c|c|}
\hline Descripción & Cantidad & Unidad \\
\hline Caudal & 0,021 & $\mathrm{~m}^{3} / \mathrm{s}$ \\
\hline Velocidad & 0,292 & $\mathrm{~m} / \mathrm{s}$ \\
\hline Reynolds (Régimen turbulento) & 94178,143 & - \\
\hline Factor de fricción (f) diagrama \\
Moody & 0,0205 & - \\
\hline Factor de fricción (f) fórmula & 0,0205 & - \\
\hline Logitud de tuberia (LT) & 5500 & $\mathrm{~m}$ \\
\hline Altura 2 (h2) & 660 & $\mathrm{~m}$ \\
\hline Altura 1 (h1) & 550 & $\mathrm{~m}$ \\
\hline Pérdida en tubería (hL) & 1,611 & $\mathrm{~m}$ \\
\hline Energía transferida al agua (hA) & 111,616 & $\mathrm{~m}$ \\
\hline $\begin{array}{c}\text { Energía transferida al agua (hA) } \\
\text { pérdidas menores }\end{array}$ & 111,624 & $\mathrm{~m}$ \\
\hline Potencia requerida de la bomba & 23248,682 & $\mathrm{~W}$ \\
\hline \multirow{2}{*}{ Total de Pérdidas } & 1,695 & $\mathrm{~m}$ \\
\cline { 2 - 3 } & 0,164 & $\mathrm{~atm}$ \\
\cline { 2 - 3 } & 126,733 & $\mathrm{~atm} * \mathrm{~m}^{3}$ \\
\cline { 2 - 3 } & 12841269,611 & $\mathrm{Joules}$ \\
\cline { 2 - 3 } & 0,004 & $\mathrm{MWh}$ \\
\cline { 2 - 3 } & 3,567 & $\mathrm{kWh}$ \\
\hline
\end{tabular}

La Tabla 29 muestra los parámetros de la bomba y del bombeo inicial, donde se observa que la energía total disponible para el bombeo final es de 201167 238,233 Joules, mismo que otorga una capacidad para bombear $57,476 \mathrm{~m}^{3}$, al calcular la diferencia entre el volumen total de los tanques de almacenamiento que se observa en la Tabla 9 y la capacidad de volumen bombeado, se tiene que existe un volumen sobrante de $0,044 \mathrm{~m}^{3}$, por lo que el bombeo inicial cubre la demanda de bombear el volumen faltante por bombear de la Tabla 16.

Se observa también en la Tabla 29 que la bomba cumple los parámetros necesarios para bombeo, con una eficiencia de $60 \%$, se requieren 4 bombas colocadas en serie y se observa que la altura proporcionada por las bombas supera a la altura que se requiere vencer, además cumple con que la potencia real de la bomba es superior al cálculo de la potencia requerida de la bomba. 
Tabla 29: Parámetros de bomba y bombeo inicial

\begin{tabular}{|c|c|c|}
\hline Descripción & Cantidad & Unidad \\
\hline Energía disponible para bombeo & 96,700 & $\mathrm{kWh}$ \\
\hline $\begin{array}{l}\text { Energía disponible para bombeo - } \\
\text { pérdidas totales }\end{array}$ & 93,133 & kWh \\
\hline Eficiencia de la bomba & $60 \%$ & \\
\hline $\begin{array}{l}\text { Energía total disponible para } \\
\text { bombeo }\end{array}$ & 201167238,233 & Joules \\
\hline Volumen máximo a bombear & 57,476 & $\mathrm{~m}^{3}$ \\
\hline Volumen faltante por bombear & $-0,044$ & $\mathrm{~m}^{3}$ \\
\hline Tiempo de bombeo & 0,750 & horas \\
\hline Caudal necesario para bombeo & 0,021 & $\mathrm{~m}^{3} / \mathrm{s}$ \\
\hline Altura a vencer & 110 & $\mathrm{~m}$ \\
\hline Potencia necesaria para bombeo & 99,915 & HP \\
\hline $\begin{array}{l}\text { Altura proporcionada por la } \\
\text { bomba }\end{array}$ & 35 & $\mathrm{~m}$ \\
\hline \# bombas & 4 & $\mathrm{u}$ \\
\hline $\begin{array}{c}\text { Altura proporcionada por las } \\
\text { bombas }\end{array}$ & 140 & $\mathrm{~m}$ \\
\hline Potencia requerida de la bomba & 24,979 & $\mathrm{HP}$ \\
\hline Potencia real de la bomba & 25 & $\mathrm{HP}$ \\
\hline
\end{tabular}

Para la obtención de la ecuación de la curva resistente del sistema se utiliza el método mostrado en el punto 2.5. Selección de la(s) Bomba(s) para Retorno de Agua desde Tanques hasta Reservorio, de donde se reemplazan los datos obtenidos dejando (18) en función de $\mathrm{Q}_{\mathrm{T}}$, como se indica en (27).

$$
H_{r}=2,75 E-4 * Q_{T}{ }^{2}+110
$$

Para la obtención de la ecuación de la curva motriz de la bomba se considera el caudal calculado en la Tabla 28.

En la metodología, en el punto 2.5. Selección de la(s) Bomba(s) para Retorno de Agua desde Tanques hasta Reservorio, se tiene el procedimiento para obtener la ecuación de la(s) bomba(s), de donde se obtienen los datos que se indican en la Tabla 30, correspondientes al bombeo final.

Tabla 30: Datos ecuaciones del sistema de bombeo final

\begin{tabular}{|c|c|c|c|c|c|}
\hline $\begin{array}{c}\mathbf{Q} \\
{\left[\mathbf{m}^{\mathbf{3}} / \mathbf{h}\right]}\end{array}$ & Hr $[\mathbf{m}]$ & $\mathbf{H m}[\mathbf{m}]$ & $\begin{array}{c}\mathbf{Q} \\
{\left[\mathbf{m}^{\mathbf{3}} / \mathbf{h}\right]}\end{array}$ & $\mathbf{H r}[\mathbf{m}]$ & Hm $[\mathbf{m}]$ \\
\hline 0 & 110,000 & 137,676 & 125 & 114,302 & 140,576 \\
\hline 25 & 110,172 & 141,256 & 150 & 116,195 & 136,656 \\
\hline 50 & 110,688 & 143,336 & 175 & 118,432 & 131,236 \\
\hline 75 & 111,549 & 143,916 & 200 & 121,013 & 124,316 \\
\hline 100 & 112,753 & 142,996 & & & \\
\hline
\end{tabular}

La ecuación (28) se obtiene de multiplicar el número de bombas que corresponde a 4 , tal como indica la Tabla 29, por la ecuación (22).

$$
H_{m}=-1,2 E-3 * Q_{T}{ }^{2}+0,1732 * Q_{T}+137,68
$$

En la Tabla 31 se indican los datos del punto de funcionamiento, mismo que corresponde a $\left(\mathrm{Q}_{\mathrm{T}} ; \mathrm{H}_{\mathrm{m}}\right)$, por lo tanto, el PF es $(205,336 ; 122,649)$.

Tabla 31: Punto de funcionamiento del bombeo final

\begin{tabular}{|c|c|c|}
\hline Descripción & Cantidad & Unidad \\
\hline $\mathrm{QT}$ & 205,336 & $\mathrm{~m}^{3} / \mathrm{h}$ \\
\hline $\mathrm{Hr}$ & 122,649 & $\mathrm{~m}$ \\
\hline $\mathrm{Hm}$ & 122,649 & $\mathrm{~m}$ \\
\hline $\mathrm{Hr}-\mathrm{Hm}$ & 0,000 & $\mathrm{~m}$ \\
\hline
\end{tabular}

\subsection{Uso de sistemas de energía híbridos}

En el caso de la energía eólica se debe buscar la forma de que esta pueda ser almacenada, ya que en ocasiones la potencia disponible del viento supera la demanda requerida y por lo tanto esa energía se desperdicia [6]. La energía generada por los sistemas híbridos de bajo alcance debe transmitirse para su utilización en los lugares no muy alejados de la fuente, porque la transmisión a larga distancia es problemática debido a la potencia eléctrica que es proporcional al producto de la corriente y la tensión [2]. En el caso planteado, se observa que la colocación de reservorios intermedios permite aprovechar la energía disponible en las zonas donde no es posible operar con toda la capacidad instalada de generación.

\section{CONCLUSIONES Y RECOMENDACIONES}

Teniendo en cuenta las diferentes ubicaciones de los estudios investigados que se muestran en la Tabla 1, cabe indicar que en ninguno de ellos se menciona un aspecto tan importante como el que trae consigo el nombre de las Islas Galápagos, debido a la importancia natural que estas tienen para la preservación del ecosistema del planeta.

Se considera que lo más viable es tener un reservorio superior de agua en una ubicación alta y mejor aún que haya sido formado naturalmente, capaz de poder obtener un caudal adecuado para generación hidroeléctrica, reduciendo el impacto con la sola implementación de un reservorio inferior en una zona que no afecte de manera sustancial el paisaje.

En cuanto a la generación eólica se observa el desaprovechamiento de dicho recurso, en vista de que después de hacer el análisis de la velocidad del viento durante el año 2018, se concluye que se estaría perdiendo en promedio cerca de $1200 \mathrm{kWh}$, tal como se muestra en la Generación perdida de la Tabla 6.

De acuerdo al recurso hídrico de la laguna El Junco y al análisis de generación, se concluye que existe plena posibilidad de cubrir la demanda del pico de la noche de San Cristóbal, correspondiente a 715,45 kWh de energía, sin necesidad de hacer uso de MCI.

Debido a la energía existente entre la demanda de la isla y la aerogeneración esperada, se concluye que existe plena posibilidad de hacer uso de la energía sobrante en el lapso de tiempo de 01:10h hasta 07:05h donde la demanda baja a tal punto que existe un excedente de aerogeneración que estaría siendo desaprovechado, y que puede usarse para el propósito del almacenamiento por bombeo que se propone en esta investigación.

Se utilizan rectángulos para el análisis de la energía de bombeo debido a que este debe ser constante en el tiempo para que entren en funcionamiento las bombas necesarias en cada etapa de bombeo. 
El bombeo intermedio es el principal, ya que este es el que transporta la mayor cantidad de volumen de agua de vuelta hacia la laguna El Junco, con el uso de 11 bombas y corresponde a 714,869 $\mathrm{m}^{3}$, sin embargo, se contempla el uso de otras dos etapas de bombeo que son capaces de bombear un volumen faltante que corresponde a $57,432 \mathrm{~m}^{3}$, tanto antes como después del bombeo intermedio.

Se contempla un tanque de almacenamiento intermedio que se calcula en base al volumen faltante que no es capaz de bombear la energía producida en el bombeo intermedio, el bombeo de dicho volumen se cubre plenamente con las otras dos etapas, del total de 11 bombas, 7 de estas son utilizadas para un bombeo inicial de $72,011 \mathrm{~m}^{3}$ de agua, y las 4 bombas restantes son utilizadas para un bombeo final de $57,476 \mathrm{~m}^{3}$ de agua.

Las bombas son capaces de entregar un mayor caudal según el PF que se obtiene con las gráficas de la curva del sistema de bombas y de la curva resistente del sistema para cada etapa de bombeo, sin dejar de cumplir con la altura requerida para lograr dicho bombeo.

Se concluye que el almacenamiento por bombeo es una muy buena opción para los proyectos híbridos, debido a que, de los cinco sistemas híbridos investigados que se muestran en la Tabla 1, dos de estos ya se encuentran con dicho sistema, otros dos se encuentran con proyección de implementarlo, y tan solo uno, no lo tiene debido a que este es el único proyecto que no es dentro de una isla.

Se debe considerar que se plantee un estudio similar para otras islas como Santa Cruz, Isabella, Floreana, y otras islas a nivel mundial, debido a que el almacenamiento por bombeo es preferido para un sistema híbrido ubicado en islas, y mejor aún si tienen potencial energético renovable.

\section{REFERENCIAS BIBLIOGRÁFICAS}

[1] B. Dursun and B. Alboyaci, "The contribution of wind-hydro pumped storage systems in meeting Turkey's electric energy demand," Renewable and Sustainable Energy Reviews, vol. 14, pp. 19791988, 2010.

[2] P. R. Naskar and S. Bondyopadhyay, "Hybrid renewable energy: future of the world," in IOP Conference Series: Materials Science and Engineering, 2018, p. 012073.

[3] T. Ma, H. Yang, L. Lu, and J. Peng, "Technical feasibility study on a standalone hybrid solar-wind system with pumped hydro storage for a remote island in Hong Kong," Renewable energy, vol. 69, pp. 7-15, 2014.

[4] Y. Saad, C. Nohra, R. Younes, S. Abboudi, A. Ilinca, H. Ibrahim, et al., "Study of an optimized winddiesel hybrid system for canadian remote sites," in
Electrical Power and Energy Conference (EPEC), 2017 IEEE, 2017, pp. 1-6.

[5] M. A. Cruz, M. E. Segatto, I. Yahyaoui, A. Atieh, and H. R. Rocha, "Feasibility analysis of a wind pumped hydroelectric storage system on the Island of Brava," in Renewable Energy Congress (IREC), 2018 9th International, 2018, pp. 1-6.

[6] Y. Saad, R. Younes, S. Abboudi, and A. Ilinca, "Hydro-pneumatic storage for wind-diesel electricity generation in remote sites," Applied Energy, vol. 231, pp. 1159-1178, 2018.

[7] U. S. Energy Information Administration, U.S. Department of Energy, Sargent \& Lundy, "Capital Cost and Performance Characteristic Estimates for Utility Scale Electric Power Generating Technologies," Independent Statistics \& Analysis, 2020.

[8] L. Bayón, J. Grau, M. Ruiz, and P. J. E. Suárez, "A comparative economic study of two configurations of hydro-wind power plants," vol. 112, pp. 8-16, 2016.

[9] S. Phommixay, M. L. Doumbia, and D. L. St-Pierre, "Optimal economic operation strategy of wind turbine-diesel unit with pumped hydro energy storage," in Ecological Vehicles and Renewable Energies (EVER), 2018 Thirteenth International Conference on, 2018, pp. 1-6.

[10] R. Hemmati, "Optimal cogeneration and scheduling of hybrid hydro-thermal-wind-solar system incorporating energy storage systems," Journal of Renewable and Sustainable Energy, vol. 10, p. 014102, 2018.

[11] M. Bianchi, L. Branchini, N. Cavina, A. Cerofolini, E. Corti, A. De Pascale, et al., "Managing wind variability with pumped hydro storage and gas turbines," vol. 45, pp. 22-31, 2014.

[12] R. Indhumathi, E. T. R. Smiline, and V. Rajaa, "Hybrid System with Dynamic Voltage Restorer for Power Quality Improvement," Journal of Chemical and Pharmaceutical Sciences ISSN, vol. 974, p. $2115,2017$.

[13]A. G. Tsikalakis, Y. A. Katsigiannis, E. S. Karapidakis, and K. E. Fiorentzis, "Evaluating the effect of wind-hydro hybrid power stations on the operation of Cretan power system," in Universities Power Engineering Conference (UPEC), 2017 52nd International, 2017, pp. 1-6.

[14]F. Kose and M. N. Kaya, "Analysis on meeting the electric energy demand of an active plant with a wind-hydro hybrid power station in Konya, Turkey: Konya water treatment plant," Renewable energy, vol. 55, pp. 196-201, 2013. 
[15]P. A. Colinvaux and E. K. Schofield, "Historical ecology in the Galapagos Islands: I. A Holocene pollen record from El Junco Lake, Isla San Cristobal," The Journal of Ecology, pp. 989-1012, 1976.

[16]C. Brumann, "Shifting tides of world-making in the UNESCO World Heritage Convention: Cosmopolitanisms colliding," Ethnic and Racial Studies, vol. 37, pp. 2176-2192, 2014.

[17]A. K. Dey, "Hybrid Hydro Renewable Energy Storage Model," in IOP Conference Series: Materials Science and Engineering, 2018, p. 012046.

[18]Á. B. Villa Caigua, "Modelos de Zonificación utilizando Información Geoespacial a través de SIG, para establecer Categorías de Manejo en función de los conflictos de la reserva hídrica y ecológica de San Cristóbal Galápagos," Quito: USFQ, 2016, 2016.

[19] M. Steinitz-Kannan, M. A. Riedinger, W. Last, M. Brenner, and M. C. Miller, "Un registro de 6000 años de manifestaciones intensas del Fenómeno de El Niño en sedimentos de lagunas de las islas Galápagos," Bulletin de l'Institut français d'études andines, vol. 27, 1998.

[20]Á. G. P. Palacios, S. A. I. Rodríguez, E. D. V. Fuentes, V. M. C. Quinto, N. L. M. Párraga, and F. E. Z. Gavilanes, "Producción de energía eólica en Ecuador," Ciencia Digital, vol. 3, pp. 22-32, 2019.

[21]M. C. Solís Gallo, "Análisis de los costos y los beneficios económicos de la sustitución de combustibles fósiles por energía renovable para la generación de energía eléctrica. Caso: Proyecto eólico de San Cristobal. Período 2007-2009," QUITO/PUCE/2011, 2011.

[22]R. L. Mott, Mecanica de Fluidos 6/e: Pearson educación, 2006.

[23]R. V. Marbello Pérez, "Bombas hidráulicas rotodinámicas: teoría y aplicaciones," Escuela de Geociencias y Medio Ambiente, 2007.

[24] G. H. D. Libanori, V. d. C. N. Pinheiro, and A. L. J. I. J. o. E. R. Francato, "Hybrid power plants as an alternative to fit pumped-storage hydro in Brazilian electricity sector regulatory framework," vol. 42, pp. 4898-4908, 2018.

[25] G. López Álvarez, "Planificación de sistemas de almacenamiento para la integración óptima de renovables en sistemas eléctricos," 2017.

[26] G. Al Zohbi, P. Hendrick, C. Renier, and P. Bouillard, "The contribution of wind-hydro pumped storage systems in meeting Lebanon's electricity demand," International Journal of Hydrogen Energy, vol. 41, pp. 6996-7004, 2016.
[27]C. Casarotto, J. Romano, and C. Collihuin, "EVALUACIÓN DE SISTEMAS HÍBRIDOS PARA ELECTRIFICACIÓN DE ZONAS REMOTAS MEDIANTE HOMER," in Universidad Nacional de Comahue. Ponencia. Cuarto Congreso Nacional-Tercer Congreso Iberoamericano Hidrogeno y Fuentes Sustentables de Energía, 2011.

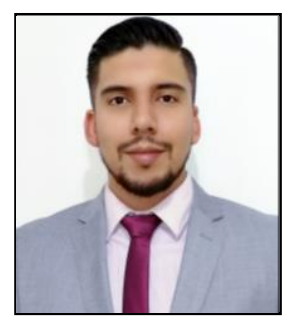

Vladimir Ernesto Burbano.Nació en Tulcán, Ecuador en 1989. Recibió su título de Ingeniero en Mecatrónica de la Universidad de las Fuerzas Armadas ESPE en 2015; y su título de Máster en Ecoeficiencia Industrial con mención en Eficiencia Energética de la Universidad Internacional SEK del Ecuador en 2020. Su campo de investigación se encuentra relacionado con la Eficiencia Energética.

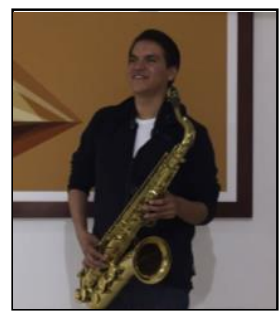

Ricardo Andrés Narváez.- Nació en Quito, Ecuador en 1986. Recibió su título de Ingeniero Químico de la Universidad Central del Ecuador en 2009; de Máster en Energías Renovables de la Universidad de Loughborough, Leicestershire, de UK en 2012; y su título de Doctor en la Universidad Loughborough, en 2019. Sus campos de investigación están relacionados con las Energías Renovables y la Eficiencia Energética. 